
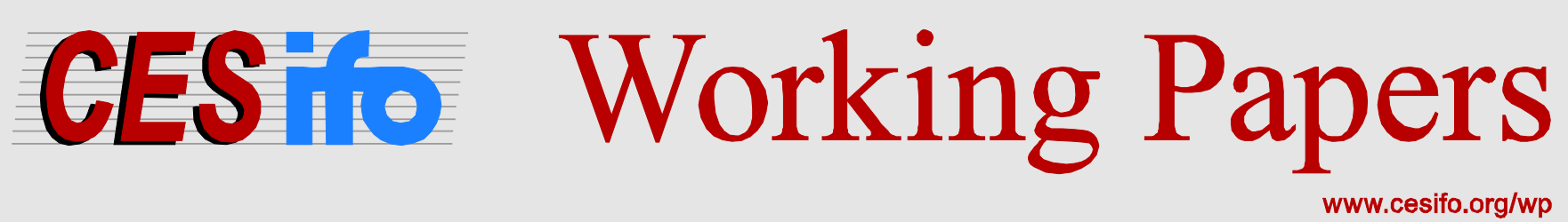

\title{
A Non-linear Forecast Combination Procedure for Binary Outcomes
}

\author{
Kajal Lahiri \\ Liu Yang
}

\author{
CESIFO WORKING PAPER NO. 5175 \\ CATEGORY 12: EMPIRICAL AND THEORETICAL METHODS \\ JANUARY 2015
}

ISSN 2364-1428

An electronic version of the paper may be downloaded

- from the SSRN website:

- from the RePEc website:

- from the CESifo website:

wWw.SSRN.com

Www.RePEc.org

www.CESifo-group.org/wp

\section{CESifo}




\title{
A Non-linear Forecast Combination Procedure for Binary Outcomes
}

\begin{abstract}
We develop a non-linear forecast combination rule based on copulas that incorporate the dynamic interaction between individual predictors. This approach is optimal in the sense that the resulting combined forecast produces the highest discriminatory power as measured by the receiver operating characteristic (ROC) curve. Under additional assumptions, this rule is shown to be equivalent to the quintessential linear combination scheme. To illustrate its usefulness, we apply this methodology to optimally aggregate two currently used leading indicators - the ISM new order diffusion index and the yield curve spread - to predict economic recessions in the United States. We also examine the sources of forecasting gains using a counterfactual experimental set up.
\end{abstract}

JEL-Code: C110, C150, C380, C530, E370.

Keywords: receiver operating characteristic curve, Copula, Bayesian methods, Markov chain Monte Carlo, yield spread, ISM diffusion index.

Kajal Lahiri

Department of Economics

University at Albany, SUNY

USA - NY 12222

klahiri@albany.edu
Liu Yang*

Business School

Nanjing University

P.R. China - Jiangsu 210093

lyang2@albany.edu

*corresponding author

The authors are grateful to James Ramsey and other participants at the 22nd SNDE Symposium in New York city, to Joerg Breitung, Frank Diebold, Hans Manner, Ataman Ozyildirim, Philip Rothman, Pravin Trivedi, and David Zimmer for making valuable comments and suggestions. However, we solely are responsible for any errors and omissions. 


\section{Introduction}

In economic forecasting, it is not uncommon to have multiple predictors for the single target variable of interest. Each predictor may contain independent information pertinent to the target that others do not have. Instead of focusing on the best predictor, one can achieve diversification gains from combining all of them in an appropriate manner. Bates and Granger (1969) have suggested a linear scheme to combine a set of forecasts with data-driven weights. Granger and Ramanathan (1984) showed that this linear combination method is numerically equivalent to a linear regression of the target variable on the forecasts. Timmermann (2006) provided a comprehensive overview of various combination methods.

This article proposes a non-linear combination procedure to predict a binary outcome variable. Binary events, such as loan defaults, occurrence of recessions, passage of a specific legislation, etc., are frequently involved in numerous economic decisions. The conventional combination methodologies are known to work well for continuous target variables, such as GDP growth and inflation rates. However, it is possible that they may fail to capture some important features of a binary target variable. Lahiri and Yang (2013b) highlighted the uniqueness of a binary event and summarized a large body of literature to forecast this type of target variables by considering their distinct features.

To quantify the forecast performance so that competing models can be compared, an appropriate criterion is necessary. Due to the very nature of a binary event, the joint distribution between forecasts and actuals are of special forms, which can be utilized to design a wide array of tools for forecast evaluation. In practice, the Brier score (Brier (1950)), or the mean squared error between forecasts and actuals, is by the far the most widely employed. Though useful by itself, this score is influenced by the marginal information regarding the actuals, and as a result is often viewed as a poor metric for measuring performance in many cases. For this reason, this paper uses the receiver operating characteristic (ROC) curve, which is especially designed to assess the efficacy of a forecasting system with a binary target. An attractive property of ROC curves is their insensitivity to changes in the event distribution (Fawcett (2006)). Furthermore, it completely describes the conditional distribution of the 
forecast given the actual. This graphic device was originally proposed in the signal detection theory during the 1950s, and has gained increasing popularity in applications, ranging from meteorology to medical sciences, and psychology, among other fields. The readers are referred to Krzanowski and Hand (2009), Pepe (2003), Swets et al. (2000), and Zhou et al. (2002) for a general introduction to this methodology. Recently, interest in ROC curves has grown among econometricians. Several economic and financial applications of ROC can be found in Berge and Jordà (2011), Drehmann and Juselius (2012), Lahiri and Wang (2013), and Lahiri and Yang (2013a).

Specifically, we separately model the marginal distributions of individual predictors and their dependence structure given each materialized regime of the target. The ratio of the joint densities is taken as the combination rule, which not only augments the predictive accuracy of each single predictor but is optimal within a family of all combination rules in the sense of maximizing the discriminatory power as measured by the ROC curve. The implied ROC curve of the combined forecasts has no known analytic form, a fact that makes the statistical inference inconvenient to be conducted in the standard parametric framework. To address this problem, we will develop a Bayesian variant of this model equipped with a non-informative prior. One of the appealing merits of the proposed procedure is that it allows for any plausible margin and dependence pattern in the predictors. It also nests the linear benchmark as a special case under some additional distributional assumptions. We demonstrate the effectiveness of our approach with an empirical illustration to predict the economic recessions in the United States based on two currently used leading indicators: ISM new order diffusion index and the yield spread.

This paper contributes to the literature on forecast combination in three aspects. First, we use ROC, in lieu of the usual mean squared error, as the measure of performance of the combined forecasts since the latter tends to reward hedging behavior of a forecaster when it is used to evaluate uncommon event probabilities (Stephenson (2000)). Second, an optimal decision rule to combine multiple predictors to maximize the discrimination capacity is formulated within a robust Bayesian framework. An algorithm is also given to make the rule practically operational. In addition, a counterfactual exercise is undertaken to identify several important determinants of a better forecast, which in turn suggests possible paths to follow to 
enhance the reliability of the combined forecasts in the present framework.

The rest of the paper is organized as follows. In Section 2, the Bayesian parametric model is introduced and the optimal decision rule is derived theoretically. The empirical application and the related computational issues are presented in Section 3, which is followed by a counterfactual experiment in Section 4 . Section 5 closes this paper with further remarks.

\section{ROC and non-linear combination methodology}

\subsection{A copula-based Bayesian approach}

We develop a parsimonious, yet flexible, Bayesian parametric model in this section to combine the information inherent in $I$ predictors for a binary event. Throughout this paper, $Z_{t}$ and $X_{i t}$ (for $i=1,2, \ldots, I$ ) denote the binary target variable and the $i$ th predictor in period $t$, respectively. We use upper case letters to denote cumulative distribution functions and corresponding lower case letters to denote the density functions.

Our Bayesian combination model consists of two elements: a conditional likelihood and a prior. We denote the joint conditional distribution of $X_{t} \equiv\left(X_{1 t}, X_{2 t}, \ldots, X_{I t}\right)$ given $Z_{t}$ by $H\left(x_{1}, x_{2}, \ldots, x_{I} \mid Z_{t}\right)$, which can be further decomposed into the marginal distribution of $X_{i t}$ given $Z_{t}$ (denoted by $F_{i}\left(x_{i} \mid Z_{t}\right)$ ) for $i=1,2, \ldots, I$ and the dependence structure among $X_{t}$ given $Z_{t}$. The latter is characterized by the copula associated with $H\left(x_{1}, x_{2}, \ldots, x_{I} \mid Z_{t}\right)$. By Sklar's theorem, for each $j \in\{0,1\}$, there exists a unique copula $C_{j}$ such that $H\left(x_{1}, x_{2}, \ldots, x_{I} \mid Z_{t}=\right.$ $j)=C_{j}\left(F_{1}\left(x_{1} \mid Z_{t}=j\right), F_{2}\left(x_{2} \mid Z_{t}=j\right), \ldots, F_{I}\left(x_{I} \mid Z_{t}=j\right)\right)$ for all $\left(x_{1}, x_{2}, \ldots, x_{I}\right) \in R^{I}$ when $X_{t}$ is a continuous random vector. Assuming $C_{j}$ is twice differentiable, the corresponding copula density is $c_{j}\left(y_{1}, y_{2}, \ldots, y_{I}\right) \equiv \frac{\partial^{I} C_{j}\left(y_{1}, y_{2}, \ldots, y_{I}\right)}{\partial y_{1} \partial y_{2} \ldots \partial y_{I}}$. In general, copulas are multivariate distribution functions whose one-dimensional marginals are uniform on the interval $(0,1)$; they enable us to construct large families of joint distributions. The popularity of copulas in empirical macroeconomics and finance owes much to their flexibility by freeing the analyst from considering only existing multivariate distributions. The stream of literature on a general introduction to the modeling strategies based on copulas includes Trivedi and Zimmer (2005), 
Nelsen (2006), and Patton (2012). Anatolyev(2009), Patton(2006) and Scotti(2011) applied this methodology to predict multiple economic events. Patton (2013) provided a recent survey on copula methods to forecasting multivariate time series.

Let $f_{i R}\left(x_{i} ; \varphi_{i R}\right)$ and $f_{i E}\left(x_{i} ; \varphi_{i E}\right)$ be the parametrized conditional densities of $X_{i t}$ given $Z_{t}=$ 1 and $Z_{t}=0$ respectively. ${ }^{1}$ The likelihood function for period $t$ can be written as

$L_{t}(\theta) \equiv \begin{cases}c_{R}\left(F_{1 R}\left(X_{1 t} ; \varphi_{1 R}\right), F_{2 R}\left(X_{2 t} ; \varphi_{2 R}\right), \ldots, F_{I R}\left(X_{I t} ; \varphi_{I R}\right) ; \gamma_{R}\right) \prod_{i=1}^{I} f_{i R}\left(X_{i t} ; \varphi_{i R}\right) & \text { if } Z_{t}=1 \\ c_{E}\left(F_{1 E}\left(X_{1 t} ; \varphi_{1 E}\right), F_{2 E}\left(X_{2 t} ; \varphi_{2 E}\right), \ldots, F_{I E}\left(X_{I t} ; \varphi_{I E}\right) ; \gamma_{E}\right) \prod_{i=1}^{I} f_{i E}\left(X_{i t} ; \varphi_{i E}\right) & \text { otherwise. }\end{cases}$

where $c_{R}\left(y_{1}, y_{2}, \ldots, y_{I} ; \gamma_{R}\right)$ and $c_{E}\left(y_{1}, y_{2}, \ldots, y_{I} ; \gamma_{E}\right)$ are the parametrized copula densities given $Z_{t}=1$ and $Z_{t}=0$ respectively. $\theta \equiv\left(\varphi_{1 R}^{\prime}, \varphi_{2 R}^{\prime}, \ldots, \varphi_{I R}^{\prime}, \varphi_{1 E}^{\prime}, \varphi_{2 E}^{\prime}, \ldots, \varphi_{I E}^{\prime}, \gamma_{R}^{\prime}, \gamma_{E}^{\prime}\right)^{\prime}$ is the parameter vector. Given a sample $X_{T} \equiv\left\{\left(Z_{t}, X_{1 t}, X_{2 t}, \ldots, X_{I t}\right): t=1, \ldots, T\right\}$, the sample likelihood function $L_{T}\left(X_{T} \mid \theta\right)$ is calculated as $\prod_{t=1}^{T} L_{t}(\theta)$. To complete the Bayesian model, a prior for $\theta$ is needed. Given a specific functional form for $L_{T}\left(X_{T} \mid \theta\right)$, it may be cumbersome, if not impossible, to construct the conjugate prior. In this section, we use a generic prior $P(\cdot)$ to express our knowledge on $\theta$ before $X_{T}$ is observed. The posterior $P\left(\theta \mid X_{T}\right)$ is derived by Bayes' theorem, that is,

$$
P\left(\theta \mid X_{T}\right)=\frac{L_{T}\left(X_{T} \mid \theta\right) P(\theta)}{\int_{\theta} L_{T}\left(X_{T} \mid \theta\right) P(\theta) d \theta} .
$$

The main goal of this paper is to compare the accuracy of forecasts based on each $X_{i t}$ with those made using all of them. A number of forecast skill measures have been proposed in the literature to quantify the performance of competing forecasts. Here, we use the receiver operating characteristic (ROC) curve and the area under the curve (AUC). ROC provides a summary of the discriminatory power of a forecasting system in distinguishing between $Z_{t}=1$ and $Z_{t}=0$. If the forecasts are completely insensitive to the value $Z_{t}$ would take, they have zero discriminatory power. On the other hand, forecasts which take one value when $Z_{t}=1$ and take another when $Z_{t}=0$ would obviously possess the highest discriminatory power. Most real-life forecasts lie between these two extremes. Suppose we predict $Z_{t}=1$

\footnotetext{
${ }^{1}$ In our empirical application in Section $3, Z_{t}=1$ if a recession occurred in month $t$. Otherwise, $Z_{t}=0$ when an expansion occurred. Hence, we use subscript "R" ("E") for $Z_{t}=1\left(Z_{t}=0\right)$.
} 
whenever $X_{1 t}$ exceeds a threshold $w$. We can define two conditional probabilities resulting from this decision rule, namely,

$$
\begin{gathered}
\zeta(w) \equiv P\left(X_{1 t}>w \mid Z_{t}=1\right)=1-F_{1 R}\left(w ; \varphi_{1 R}\right), \\
\kappa(w) \equiv P\left(X_{1 t}>w \mid Z_{t}=0\right)=1-F_{1 E}\left(w ; \varphi_{1 E}\right) .
\end{gathered}
$$

$\zeta(w)$ is referred to as the hit rate and it is the probability of correct forecast when $Z_{t}=1$. $\kappa(w)$ is called the false alarm rate or the probability of false forecast when $Z_{t}=0$. Ideally, we hope $\zeta(w)$ could be as large as possible and $\kappa(w)$ should be as small as possible. Both of them are functions of $w$. In general, given the forecasting system, it is hard to achieve a high value of $\zeta(w)$ without changing $\kappa(w)$. The tradeoff between them is depicted by plotting the pair $(\kappa(w), \zeta(w))$ in a unit square for every $w$. The resulting ROC curve is an increasing function from $(0,0)$ to $(1,1)$. The ROC curve for forecasts with zero discriminatory power is represented by the diagonal line in the unit square with its AUC 0.5. Conversely, the ROC curve described by the left and upper boundaries of the square has the highest discriminatory power with its AUC 1. Most real-life forecasts yield an ROC curve lying in the upper triangular area whose AUC is strictly between 0.5 and 1.

When multiple predictors are available, none of them could be maximally utilized unless the information contained in them is combined in an efficient manner. Suppose we only use $X_{1 t}$. We may consider predicting $Z_{t}=1$ when $X_{1 t}>\eta$ or when $\Lambda\left(X_{1 t}\right)>\eta$, where $\Lambda(\cdot)$ is a known function. Krzanowski and Hand (2009) showed that the ROC curves generated by the rules $\Lambda\left(X_{1 t}\right)>\eta$ and $X_{1 t}>\eta$ will be the same if $\Lambda(\cdot)$ is a strictly increasing function. In addition, if multiple predictors are available, it is natural to combine them for the optimal performance in the sense that the hit rate is maximized for any given false alarm rate. The region $C_{\alpha}(\eta)$ defined as

$$
C_{\alpha}(\eta) \equiv\left\{X_{t}: \frac{h\left(X_{t} \mid H_{1}\right)}{h\left(X_{t} \mid H_{0}\right)}>\eta\right\}
$$

plays a critical role in testing the null hypothesis $H_{0}: \theta=\theta_{0}$ against the alternative $H_{1}: \theta=\theta_{1}$. Here, $X_{t}=\left(X_{1 t}, X_{2 t}, \ldots, X_{I t}\right)$ is the observed data for predictors, $h\left(X_{t} \mid H_{j}\right)$ is the likelihood function under $H_{j}$ for $j=0,1$, and $\eta$ is a constant such that $P\left(X_{t} \in\right.$ 
$\left.C_{\alpha}(\eta) \mid H_{0}\right)=\alpha$. Among all tests of $H_{0}$ against $H_{1}$ with the same size $\alpha$, NeymanPearson lemma states that the power, defined as $P\left(h\left(X_{t} \mid H_{1}\right) / h\left(X_{t} \mid H_{0}\right)>\eta \mid H_{1}\right)$, achieves its maximum if we reject $H_{0}$ when $h\left(X_{t} \mid H_{1}\right) / h\left(X_{t} \mid H_{0}\right)>\eta$. Therefore, the likelihood ratio test for simple hypothesis is the uniformly most powerful. The implication of this lemma is that the rule constructed from the likelihood ratio of multiple predictors maximizes the hit rate for any given false alarm rate. This rule can be used to justify the test $H_{0}: Z_{t}=0$ against $H_{1}: Z_{t}=1$. Given the parametric specification, $h\left(X_{t} \mid Z_{t}=1\right)=c_{R}\left(F_{1 R}\left(X_{1 t} ; \varphi_{1 R}\right), F_{2 R}\left(X_{2 t} ; \varphi_{2 R}\right), \ldots, F_{I R}\left(X_{I t} ; \varphi_{I R}\right) ; \gamma_{R}\right) \prod_{i=1}^{I} f_{i R}\left(X_{i t} ; \varphi_{i R}\right)$ and $h\left(X_{t} \mid Z_{t}=0\right)=c_{E}\left(F_{1 E}\left(X_{1 t} ; \varphi_{1 E}\right), F_{2 E}\left(X_{2 t} ; \varphi_{2 E}\right), \ldots, F_{I E}\left(X_{I t} ; \varphi_{I E}\right) ; \gamma_{E}\right) \prod_{i=1}^{I} f_{i E}\left(X_{i t} ; \varphi_{i E}\right)$. Thus, the likelihood ratio for the combined forecast takes the following form,

$$
\frac{c_{R}\left(F_{1 R}\left(X_{1 t} ; \varphi_{1 R}\right), F_{2 R}\left(X_{2 t} ; \varphi_{2 R}\right), \ldots, F_{I R}\left(X_{I t} ; \varphi_{I R}\right) ; \gamma_{R}\right) \prod_{i=1}^{I} f_{i R}\left(X_{i t} ; \varphi_{i R}\right)}{c_{E}\left(F_{1 E}\left(X_{1 t} ; \varphi_{1 E}\right), F_{2 E}\left(X_{2 t} ; \varphi_{2 E}\right), \ldots, F_{I E}\left(X_{I t} ; \varphi_{I E}\right) ; \gamma_{E}\right) \prod_{i=1}^{I} f_{i E}\left(X_{i t} ; \varphi_{i E}\right)}
$$

and we predict $Z_{t}=1$ if and only if (2) exceeds $\eta$. In this case, the size is $P\left(h\left(X_{t} \mid Z_{t}=\right.\right.$ 1) $\left./ h\left(X_{t} \mid Z_{t}=0\right)>\eta \mid Z_{t}=0\right)$, while the power is $P\left(h\left(X_{t} \mid Z_{t}=1\right) / h\left(X_{t} \mid Z_{t}=0\right)>\eta \mid Z_{t}=1\right)$.

Therefore, the size corresponds to the false alarm rate $\kappa(\eta)$, while the power corresponds to the hit rate $\zeta(\eta)$. Given $\kappa(\eta), \zeta(\eta)$ is maximized among all possible combination rules based on $\left(X_{1 t}, X_{2 t}, \ldots, X_{I t}\right)$.

As a forecast combination scheme, (2) makes intuitive sense. The numerator measures how likely is that the values of $\left(X_{1 t}, X_{2 t}, \ldots, X_{I t}\right)$ are observed when $Z_{t}=1$, while the denominator is the likelihood that the very values of $\left(X_{1 t}, X_{2 t}, \ldots, X_{I t}\right)$ can be seen with $Z_{t}=0$. If the truth is $Z_{t}=1$, the numerator is expected to be higher than the denominator, and vice versa. Having interpreted (2) in this way, given a threshold value $\eta, Z_{t}=1$ should be more likely to occur and thus be predicted if the ratio of these two likelihoods exceeds $\eta$. Two special cases of (2) worth mentioning. In the first case, $X_{I t}$ is completely irrelevant in the sense that it is independent of $\left(Z_{t}, X_{1 t}, \ldots, X_{(I-1) t}\right)$. In the second case, $X_{I t}$ is a non-stochastic function of $\left(X_{1 t}, \ldots, X_{(I-1) t}\right)$ and hence is completely redundant. In both cases, (2) can be shown to depend on $\left(X_{1 t}, \ldots, X_{(I-1) t}\right)$ rather than the whole $X_{t}{ }^{2}$ That is, our optimal combination scheme

\footnotetext{
${ }^{2}$ In the first case, it follows from independence that $h\left(X_{1 t}, \ldots, X_{I t} \mid Z_{t}\right)=h\left(X_{1 t}, \ldots, X_{(I-1) t} \mid Z_{t}\right) f_{I}\left(X_{I t}\right) . f_{I}\left(X_{I t}\right)$ is the marginal density of $X_{I t}$ that does not depend on $Z_{t}$ and thus it can be cancelled out from (2). In the second case, the redundancy of $X_{I t}$ implies $h\left(X_{1 t}, \ldots, X_{I t} \mid Z_{t}\right)=h\left(X_{1 t}, \ldots, X_{(I-1) t} \mid Z_{t}\right)$.
} 
automatically ignores the irrelevant and redundant predictors if they exist. The optimality of (2) has long been noted in statistics (Neyman and Pearson (1933)) and signal detection theory (Green and Swets (1966), Egan (1975)). The application of (2) to optimally combine multiple medical classifiers was formally addressed in McIntosh and Pepe (2002).

The ROC curve corresponding to the optimal rule (2) can be obtained by calculating $\zeta(\eta)$ and $\kappa(\eta)$ for each $\eta$. Suppose $X_{1 t}$ is the only predictor available. The optimal rule says that $Z_{t}=1$ is predicted if $f_{1 R}\left(X_{1 t} ; \varphi_{1 R}\right)>\eta f_{1 E}\left(X_{1 t} ; \varphi_{1 E}\right)$. Given $\eta$,

$$
\begin{aligned}
& \zeta(\eta)=P\left(f_{1 R}\left(X_{1 t} ; \varphi_{1 R}\right)>\eta f_{1 E}\left(X_{1 t} ; \varphi_{1 E}\right) \mid Z_{t}=1\right) \\
& \kappa(\eta)=P\left(f_{1 R}\left(X_{1 t} ; \varphi_{1 R}\right)>\eta f_{1 E}\left(X_{1 t} ; \varphi_{1 E}\right) \mid Z_{t}=0\right) .
\end{aligned}
$$

Sometimes, evaluation of (3) is intractable due to the parametric assumptions imposed on $F_{1}\left(x_{1} \mid Z_{t}\right)$. Consequently, we use simulation to approximate both $\zeta(\eta)$ and $\kappa(\eta)$. For example, $\zeta(\eta)$ should be close to $\zeta^{s}(\eta) \equiv \sum_{s=1}^{S} I\left(f_{1 R}\left(h_{s} ; \varphi_{1 R}\right)>\eta f_{1 E}\left(h_{s} ; \varphi_{1 E}\right)\right) / S$ if $\left\{h_{s}: s=1, \ldots, S\right\}$ is a large sequence of draws from $f_{1 R}\left(x_{1} ; \varphi_{1 R}\right)$. Given a posterior point estimate of $\theta$, such as the posterior mean, we can get the ROC curve by plotting simulated pairs $\left(\kappa^{S}(\eta), \zeta^{s}(\eta)\right)$ evaluated at this estimate of $\theta$ over a fine grid of $\eta$. The AUC value is calculated by numerical integration of the simulated ROC curve over $[0,1]$. The same procedure can be applied when any other $X_{i t}$ is considered. Evaluating (3) for combined forecasts involves simulating random samples from multivariate copulas, and the details can be found in Trivedi and Zimmer (2005, appendix). The inference is based on the posterior distribution of AUC derived by repeating this process for each draw of $\theta$.

\subsection{Robust inference}

In standard Bayesian analysis, the posterior density $P\left(\theta \mid X_{T}\right)$ summarizes all we know about $\theta$ after observing $X_{T}$ if and only if $L_{T}\left(X_{T} \mid \theta\right)$ is correct. However, even when the joint conditional distribution $H\left(x_{1}, x_{2}, \ldots, x_{I} \mid Z_{t}\right)$ is correctly specified, dynamic misspecification in the innovations can never be ruled out. This implies that $L_{T}\left(X_{T} \mid \theta\right)$, obtained by assuming (for instance) the absence of serial correlation, is not valid and $P\left(\theta \mid X_{T}\right)$ will not reflect all in- 
formation about $\theta$ with available $X_{T}$. We do not seek to model the dynamic structure in the errors, since our analysis only requires the systematic component $H\left(x_{1}, x_{2}, \ldots, x_{I} \mid Z_{t}\right)$ be correctly specified. There is also evidence that explicitly modeling the serial correlation in the context of a Markov Switching model of leading indicators as a filter jeopardizes turning point predictions, see Lahiri and Wang (1994). Fortunately, $P\left(\theta \mid X_{T}\right)$ under this type of model misspecification can be asymptotically approximated by a multivariate normal distribution with the maximum likelihood estimator $\hat{\theta}$ as its mean and the inverse of the estimated negative Hessian matrix as its covariance (Berk (1966), Bunke and Milhaud (1998), and Geweke (2005)). However, Müller (2013) has shown that the asymptotic frequentist risk associated with the posterior inference is systematically lower if the asymptotic variance of the normal posterior is replaced by a sandwich covariance matrix, which is routinely used in obtaining robust standard errors in the frequentist approach.

Specifically, we define $s_{t}(\theta) \equiv \frac{\partial \log \left(L_{t}(\theta)\right)}{\partial \theta}, I_{T}(\theta) \equiv \hat{\operatorname{Var}}\left(\frac{1}{\sqrt{T}} \sum_{t=1}^{T} s_{t}(\theta)\right)$, and $H_{T}(\theta) \equiv$ $\frac{1}{T} \sum_{t=1}^{T} \frac{\partial s_{t}(\theta)}{\partial \theta}$. Müller's sandwich covariance matrix takes the form $\frac{1}{T} H_{T}(\hat{\theta})^{-1} I_{T}(\hat{\theta}) H_{T}(\hat{\theta})^{-1}$. Here, the "bread" is the inverse of Hessian matrix $H_{T}(\hat{\theta})^{-1}$ and the "meat" is the long run variance of the score functions $I_{T}(\hat{\theta})$, which is usually calculated as a kernel-based estimate, as in Andrews (1991) and Newey and West (1987). A number of empirical examples in Müller (2013) demonstrate that the sandwich posterior inference constitutes a pragmatic improvement for Bayesian inference in parametric models, and often implies a substantially more uncertainty about model parameters. Since the relative superiority of the artificial sandwich posterior is valid only in large samples, we will implement our procedure with and without this suggestion. To save space, we only report the results obtained from the normal posterior with sandwich covariance matrix reported in the sections to follow. Fortunately, very similar conclusions were drawn by sampling from $P\left(\theta \mid X_{T}\right)$ without the sandwich covariance matrix, suggesting dynamic misspecification may not be an issue in our example.

\subsection{Relationship with extant methods}

The likelihood rule in (2) is in the same spirit as in Graham (1996). His binary combination scheme is also built upon the likelihood of receiving a particular forecast prior to a specific 
outcome, and the reason of doing so, as argued by him, is that $h\left(X_{t} \mid Z_{t}\right)$ contains information about the discrimination capacity of each predictor, as well as the correlation between predictors. However, Graham (1996) only considered combining binary forecasts, that is, each variable in $X_{t}$ is binary. Though Graham (1996) applied this rule to combine probability forecasts as well, he rounded the probability into a binary format by choosing a threshold, which would lead to information loss. The likelihood ratio rule in (2) is more general in that both continuous and discrete predictors are allowed. In addition, we established the optimality of (2) in terms of maximizing the discriminatory power, while it is not clear if the binary combination scheme in Graham (1996) is optimal in some sense.

It is interesting to note that some popular forecast combination rules can be shown to be special cases of (2). According to Bayes' theorem,

$$
\begin{aligned}
P\left(Z_{t}=1 \mid X_{t}\right) & =\frac{h\left(X_{t} \mid Z_{t}=1\right) P\left(Z_{t}=1\right)}{h\left(X_{t} \mid Z_{t}=0\right) P\left(Z_{t}=0\right)+h\left(X_{t} \mid Z_{t}=1\right) P\left(Z_{t}=1\right)} \\
& =\frac{\frac{h\left(X_{t} \mid Z_{t}=1\right)}{h\left(X_{t} \mid Z_{t}=0\right)} P\left(Z_{t}=1\right)}{P\left(Z_{t}=0\right)+\frac{h\left(X_{t} \mid Z_{t}=1\right)}{h\left(X_{t} \mid Z_{t}=0\right)} P\left(Z_{t}=1\right)}
\end{aligned}
$$

If $h\left(X_{t} \mid Z_{t}=1\right)$ and $h\left(X_{t} \mid Z_{t}=0\right)$ are multivariate normal, it follows that the conditional $\log$ odds ratio $\ln \left(\frac{P\left(Z_{t}=1 \mid X_{t}\right)}{P\left(Z_{t}=0 \mid X_{t}\right)}\right)$ is equal to

$$
\begin{aligned}
& \ln \left(\frac{P\left(Z_{t}=1\right)}{P\left(Z_{t}=0\right)}\right)+\frac{1}{2}\left(\ln \left|\Sigma_{0}\right|-\ln \left|\Sigma_{1}\right|\right)+\frac{1}{2}\left(\left(X_{t}-\mu_{0}\right)^{\prime} \Sigma_{0}^{-1}\left(X_{t}-\mu_{0}\right)-\left(X_{t}-\mu_{1}\right)^{\prime} \Sigma_{1}^{-1}\left(X_{t}-\mu_{1}\right)\right) \\
= & \ln \left(\frac{P\left(Z_{t}=1\right)}{P\left(Z_{t}=0\right)}\right)+\frac{1}{2}\left(\ln \left|\Sigma_{0}\right|-\ln \left|\Sigma_{1}\right|\right)+\frac{1}{2}\left(X_{t}^{\prime} \Sigma_{0}^{-1} X_{t}-X_{t}^{\prime} \Sigma_{1}^{-1} X_{t}-2 X_{t}^{\prime} \Sigma_{0}^{-1} \mu_{0}\right. \\
& \left.+2 X_{t}^{\prime} \Sigma_{1}^{-1} \mu_{1}+\mu_{0}^{\prime} \Sigma_{0}^{-1} \mu_{0}-\mu_{1}^{\prime} \Sigma_{1}^{-1} \mu_{1}\right) \\
= & A_{0}+A_{1}^{\prime} X_{t}+X_{t}^{\prime} A_{2} X_{t},
\end{aligned}
$$

where $\mu_{j}$ is the mean of $h\left(X_{t} \mid Z_{t}=j\right)$, and $\Sigma_{j}$ is the covariance matrix of $h\left(X_{t} \mid Z_{t}=j\right)$. In (5), $A_{0}=\ln \left(\frac{P\left(Z_{t}=1\right)}{P\left(Z_{t}=0\right)}\right)+\frac{1}{2}\left(\ln \left|\Sigma_{0}\right|-\ln \left|\Sigma_{1}\right|\right)+\frac{1}{2}\left(\mu_{0}^{\prime} \Sigma_{0}^{-1} \mu_{0}-\mu_{1}^{\prime} \Sigma_{1}^{-1} \mu_{1}\right), A_{1}=\Sigma_{1}^{-1} \mu_{1}-\Sigma_{0}^{-1} \mu_{0}$, and $A_{2}=\Sigma_{0}^{-1}-\Sigma_{1}^{-1}$. As a result,

$$
p_{t} \equiv P\left(Z_{t}=1 \mid X_{t}\right)=\frac{\exp \left(A_{0}+A_{1}^{\prime} X_{t}+X_{t}^{\prime} A_{2} X_{t}\right)}{1+\exp \left(A_{0}+A_{1}^{\prime} X_{t}+X_{t}^{\prime} A_{2} X_{t}\right)}
$$


which implies that $P\left(Z_{t}=1 \mid X_{t}\right)$, as a regression function, is consistent with a logit specification with quadratic index $A_{0}+A_{1}^{\prime} X_{t}+X_{t}^{\prime} A_{2} X_{t} \cdot{ }^{3}$ Since (2) is a strictly increasing transformation of (6), both would yield identical ROC curves. Note that $\Sigma_{1}=\Sigma_{0}$ implies $A_{2}=0$, and then $P\left(Z_{t}=1 \mid X_{t}\right)$ reduces to the regular logit specification with linear index $A_{0}+A_{1}^{\prime} X_{t}$, which is the dichotomous combination rule introduced by Kamstra and Kennedy (1998). This relationship offers insights into effectiveness of the logit regression as a type of non-linear combination scheme. Although Kamstra and Kennedy (1998) used this approach as an alternative means of combining qualitative forecasts, we have shown that it is indeed optimal under certain simplifying assumptions including $\Sigma_{1}=\Sigma_{0}$. The implication of (6) is that one can regress $Z_{t}$ on a constant, $X_{1 t}, X_{2 t}, \ldots, X_{I t}, X_{1 t}^{2}, X_{2 t}^{2}, \ldots, X_{I t}^{2}$ and all of the interaction terms like $X_{i t} X_{j t}$ using the logit model, get the maximum likelihood estimates for $A_{0}, A_{1}$ and $A_{2}$, and take the fitted probability $P\left(Z_{t}=1 \mid X_{t}\right)$ as the combined forecast. Following the same approach as in Ramsey (1969), the appropriateness of non-linearity can be assessed by examining the size and statistical significance of estimates of $A_{2}$. If the linear index is adequate, the joint test should be insignificant. If the test is significant, the non-linear combination scheme in (6) should be used. However, normality assumption is essential for the equivalence between (2) and (6) to hold.

Another popular option is the linear combination method proposed by Bates and Granger (1969), which often serves as a useful benchmark in practice. Given the probabilities generated by each individual predictor, $P\left(Z_{t}=1 \mid X_{i t}\right)$ for $i=1,2, \ldots, I$, all of which may be perfectly calibrated in the sense of Dawid $(1984)^{4}$, the linearly combined forecast can be constructed by taking the linear pool, that is,

$$
q_{t} \equiv \omega_{1} P\left(Z_{t}=1 \mid X_{1 t}\right)+\omega_{2} P\left(Z_{t}=1 \mid X_{2 t}\right)+\ldots+\omega_{I} P\left(Z_{t}=1 \mid X_{I t}\right)
$$

where $\omega_{i} \in(0,1)$ is a properly selected weight attached to $P\left(Z_{t}=1 \mid X_{i t}\right)$, and $\sum_{i=1}^{I} \omega_{i}=1$.

\footnotetext{
${ }^{3}(6)$ is a well-known result in discriminant analysis. Its derivation can be also found in Hastie et al. (2001).

${ }^{4} \mathrm{~A}$ perfectly calibrated probability forecast implies the actual frequency of the event $Z=1$ given each forecast value $p$ should be equal to the forecast itself. Formally, if $P(Z=1 \mid p)=p$, the probability forecast $p$ is said to be perfectly calibrated. Calibration and discriminatory power (as measured by the ROC curve) are two forecast skill metrics. They complement each other and measure the quality of a forecasting system from different perspectives. They can be derived from two alternative factorizations of the joint distribution between forecast and actual. See Lahiri and Yang (2013b) for more details.
} 
De Luca and Carfora (2014) provided an example where $q_{t}$ in (7) is used to combine two binary regression models for predicting recessions. It is interesting to note that the difference between (6) and (7). Ranjan and Gneiting (2010) proved that (7) will lack calibration even though each $P\left(Z_{t}=1 \mid X_{i t}\right)$ is calibrated. However, by construction, (6) is calibrated. Moreover, for every strictly proper scoring rule S defined by Gneiting and Raftery (2007), such as the usual mean squared error, $E\left(S\left(p_{t}, Z_{t}\right)\right)<E\left(S\left(q_{t}, Z_{t}\right)\right)$, which can be shown by observing that

$$
\begin{aligned}
E\left(S\left(p_{t}, Z_{t}\right)\right) & =E\left(E\left(S\left(p_{t}, Z_{t}\right) \mid X_{t}\right)\right) \\
& =E\left(p_{t} S\left(p_{t}, 1\right)+\left(1-p_{t}\right) S\left(p_{t}, 0\right)\right) \\
& <E\left(p_{t} S\left(q_{t}, 1\right)+\left(1-p_{t}\right) S\left(q_{t}, 0\right)\right) \\
& =E\left(E\left(S\left(q_{t}, Z_{t}\right) \mid X_{t}\right)\right) \\
& =E\left(S\left(q_{t}, Z_{t}\right)\right)
\end{aligned}
$$

where the first and the last equalities follow from the law of iterated expectations, the second and the fourth equalities are obtained by noting that $Z_{t}$ is a $0-1$ binary variable with $p_{t}=P\left(Z_{t}=1 \mid X_{t}\right)$, and the inequality uses the property of the strictly proper scoring rule. ${ }^{5}$ Therefore, (6) outperforms (7) in a general sense. As (2) and (6) share the same ROC curve, (6) is also preferred in terms of the discriminatory capacity as measured by ROC curve. The bottom line is (6) is always superior to (7) regardless of the optimality criterion used.

\footnotetext{
${ }^{5}$ A scoring rule assigns a numerical score, $S(p, Z)$, to the probability forecast $p$ and the binary variable $Z$. The scoring rule is negatively oriented, that is, the smaller the better. $S(p, Z)$ is strictly proper if $p S(p, 1)+(1-$ p) $S(p, 0)<p S(q, 1)+(1-p) S(q, 0)$ for all $0 \leq q \neq p \leq 1$, that is, it encourages honesty. More details on the proper scoring rule can be found in Gneiting and Raftery (2007) and Schervish (1989).
} 


\section{Application to recession prediction}

\subsection{Data description}

In this section, we will present an empirical illustration to showcase the usefulness of our methodology with $I=2$. The task is to predict future U.S. economic recessions. The monthly data we use consists of 629 observations on two leading indicators for U.S. recessions-ISM diffusion index of new orders and the yield spread—from August 1959 to December 2011 (cf. Levanon et al. (2011)). The binary target event $Z_{t}$ is the recession indicator that is one, if the recession occurred, and zero otherwise. The sample proportion of months that were in recession over this period is about $14.8 \%$, indicating that it is a relatively uncommon event. The first predictor $X_{1 t}$ is the manufacturing new orders diffusion index compiled by the Institute for Supply Management (ISM), which reflects the number of manufacturers reporting decreased orders during the previous month compared to the number reporting increased orders. A higher value of this index tends to signify a future economic recession. The yield spread, as the second predictor $X_{2 t}$, is defined as the difference between the constant maturity yields on a 3-month T-bill and the 10-year Treasury note. Previous literature has found that the yield spread is the single indicator which has the highest predictive power in terms of forecasting economic recessions at the fourth quarter horizon. There are many possible reasons why this is the case. See Estrella and Mishkin $(1996,1998)$ for comprehensive explanations. For the purpose of exposition, we use these two indicators to predict recessions six and nine months ahead.

\subsection{Model specification and computational details}

For simplicity, we consider a binormal specification, in which all marginal distributions, including $F_{1}\left(x_{1} \mid Z_{t}\right)$ and $F_{2}\left(x_{2} \mid Z_{t}\right)$, are assumed to be normal with different means and vari-

ances. Let $\mu_{R, I S M}$ and $\sigma_{R, I S M}^{2}$ be the conditional mean and variance of ISM diffusion index given $Z_{t}=1$. Likewise $\mu_{R, Y S}$ and $\sigma_{R, Y S}^{2}$ are the corresponding parameters for yield spread. 
Thus, $\varphi_{1 R}=\left(\mu_{R, I S M}, \sigma_{R, I S M}^{2}\right)^{\prime}$ and $\varphi_{2 R}=\left(\mu_{R, Y S}, \sigma_{R, Y S}^{2}\right)^{\prime} . \varphi_{1 E}$ and $\varphi_{2 E}$ are defined analogously for $Z_{t}=0$.

It may be natural to use the bivariate normal distributions for $H\left(x_{1}, x_{2} \mid Z_{t}=1\right)$ and $H\left(x_{1}, x_{2} \mid Z_{t}=0\right)$. After all, each marginal distribution is normal. This amounts to using Gaussian copulas to describe the dependence pattern between two predictors. Despite its parsimony and familiarity, the Gaussian copula has its own pitfall in that the dependence structure is restricted. In our application, we employ the copula corresponding to the bivariate t-distribution with two parameters: correlation coefficient $\rho$ and degrees of freedom $d f$. Therefore, $\gamma_{R}=\left(\rho_{R}, d f_{R}\right)^{\prime}$ and $\gamma_{E}=\left(\rho_{E}, d f_{E}\right)^{\prime}$. The t-copula is often used to model the dependence structure among returns of multiple financial assets (see for example Mashal et al. (2003) and Breymann et al. (2003)). Gaussian copula and t-copula belong to the so-called elliptical class. The correlation coefficient in both copulas captures the dependence between two random variables, say $X_{1}$ and $X_{2}$, in the center of their distributions, while the degrees of freedom in the t-copula controls for the tail dependence. The upper tail dependence coefficient $\lambda_{u}$ is defined as $\lim _{q \rightarrow 1^{-}} P\left(X_{2}>F_{2}^{-1}(q) \mid X_{1}>F_{1}^{-1}(q)\right)$, and the lower tail dependence coefficient $\lambda_{l}$ is $\lim _{q \rightarrow 0^{+}} P\left(X_{2} \leq F_{2}^{-1}(q) \mid X_{1} \leq F_{1}^{-1}(q)\right)$, where $F_{i}^{-1}(\cdot)$ is the quantile function of $X_{i}$. They measure the dependence between $X_{1}$ and $X_{2}$ when both are extremely large (small). A strong dependence in the center does not necessarily translate into the strong dependence in both tails. For instance, $\lambda_{u}=\lambda_{l}=0$ for any Gaussian copula without perfect correlation. As a result, the Gaussian copula does not entail positive dependence in both tails of distribution so that extreme events appear to be uncorrelated. However, both $\lambda_{u}$ and $\lambda_{l}$ are positive for the t-copula (Demarta and McNeil (2005)). In general, the degrees of freedom control how quickly the tail dependence shrinks towards zero. When the degrees of freedom approach infinity, the tail dependence vanishes. In this sense, the t-copula is more general. However, it requires symmetric dependence, namely, $\lambda_{u}=\lambda_{l}$, which may be too restrictive in some cases. To overcome this drawback, Demarta and McNeil (2005) has constructed t-copulas with asymmetric tail behaviors by introducing more parameters. Besides the elliptical class, there exist other flexible copulas, some of which may be more than or as flexible as t-copula, including many members within the Archimedean family. The specific choice of copulas crucially rests on the empirical setting. In our circumstance, we favor t-copula 
because it captures the dependence structure both in the center and tails of the distributions in a relatively parsimonious fashion. This can be seen from Figure 1, which presents the scatter plots of $F_{1}\left(X_{1 t} \mid Z_{t}\right)$ and $F_{2}\left(X_{2 t} \mid Z_{t}\right)$, the CDF-scaled predictors for each combination of regimes and horizons. A number of observations concentrate around the lower left and upper right corners, indicating the existence of positive tail dependence during expansion. We have plotted five contours of bivariate densities of the fitted t-copulas, with all points on a contour representing the same density value. The inner contours represent higher densities. Our framework can accommodate virtually all reasonable copulas, provided some mild regularity conditions, like smoothness, are met.

The prior $P(\cdot)$ is specified as normal. To this end, all parameters with limited range are rescaled by suitable transformations. For example, we impose a prior on $\log \left(\sigma_{R, Y S}^{2}\right)$ rather than $\sigma_{R, Y S}^{2}$. To be more specific, the mean of $P(\cdot)$ is 0 and the variance is a constant multiple $(v)$ of the sandwich covariance matrix associated with $\hat{\theta}$. In order for the prior to be relatively flat to represent the vague information, $v$ must be a large positive number. Here, we choose $v=1,000$ since the empirical results are nearly the same for any $v$ higher than 1,000 . In this case, the assumed value of the mean vector is of little relevance.

Simulating from the sandwich posterior is straightforward as long as the quasi-maximum likelihood estimator $\hat{\theta}$ is available. The attractive feature of copula facilitates the numerical computation substantially in that we can use a sequential procedure to get a preliminary estimator. In the first step, the parameters in marginal distributions $\left(\varphi_{1 R}^{\prime}, \varphi_{2 R}^{\prime}, \varphi_{1 E}^{\prime}, \varphi_{2 E}^{\prime}\right)^{\prime}$ are estimated. The dependence parameters $\left(\gamma_{R}^{\prime}, \gamma_{E}^{\prime}\right)^{\prime}$ are estimated in the second step after the estimated marginal distributions have been substituted into $L_{T}\left(X_{T} \mid \theta\right)$. Finally, we set the initial values of $\theta$ to be those obtained in the first two steps and then maximize $L_{T}\left(X_{T} \mid \theta\right)$ again to get $\hat{\theta}$. The long run covariance matrix of the score functions is estimated via quadratic spectral kernel after the data is filtered by the AR(1) prewhitening procedure as advocated by Andrews and Monahan (1992).

A convenient way of simulating from $P\left(\theta \mid X_{T}\right)$ is by using Markov chain Monte Carlo (MCMC) method. In Bayesian statistics, the Gibbs sampler is commonly used for posterior simulation when $P\left(\theta \mid X_{T}\right)$ can be easily decomposed into several conditional posterior distributions with known functional forms. The posterior draws of $\theta$ can be obtained by se- 
quentially simulating from these conditional distributions. The problem with this algorithm is that sometimes it is difficult to find such blocks of $\theta$ with closed form conditional distributions. This is the case in the current scenario since t-copulas are involved in $L_{T}\left(X_{T} \mid \theta\right)$. Thus, we consider the random walk chain constructed by a Metropolis-Hastings algorithm. Unlike Gibbs sampler, it constitutes a quite general class of posterior simulators. Specifically, suppose a draw $\theta^{b}$ in step $b$ is given. A candidate draw $\theta^{*}$ is sampled from $\theta^{b}+c N$, where $N$ is a multivariate normal vector with mean zero and variance $V$, and $c$ is a positive scale number. For estimates reported below, we use the sandwich covariance matrix as $V$. $\theta^{*}$ is accepted as the next draw $\theta^{b+1}$ if it lies within the area of higher posterior density relative to $\theta^{b}$. The details can be found in Koop (2003). The value of $c$ is determined in such a way that the resulting acceptance rate is about 25\%, as suggested by Albert (2009). The first 10,000 draws are discarded to remove the impact of the initial value. A variety of diagnostic plots and formal statistical tests based on the remaining 90,000 draws are not reported here due to brevity. All of them tend to indicate successful convergence of the chain towards $P\left(\theta \mid X_{T}\right)$.

\subsection{Empirical results}

Table 1 presents the posterior mean of $\theta$ together with two bounds of the highest posterior density (HPD) interval. This is the parameter interval of a given size in which any point delivers a higher posterior density than an arbitrary point outside the interval. It has lower and upper bounds if the posterior is unimodal. Here, the nominal size is fixed at $95 \%$. Analogous to the role a confidence interval plays in a frequentist framework, an HPD interval accounts for the uncertainty associated with a point estimate of $\theta$.

From this table, we see that $\left|\mu_{R, I S M}-\mu_{E, I S M}\right|$ shrinks towards zero as the forecast horizon goes from 6 to 9 months. This is just opposite with the yield spread. On the other hand, the yield spread tends to be more volatile than the diffusion index although the volatility of the former decreases with horizon. The two conditional distributions for the diffusion index $\left(f_{1}\left(x_{1} \mid Z_{t}=1\right)\right.$ and $\left.f_{1}\left(x_{1} \mid Z_{t}=0\right)\right)$ and the yield spread $\left(f_{2}\left(x_{2} \mid Z_{t}=1\right)\right.$ and $f_{2}\left(x_{2} \mid Z_{t}=\right.$ $0)$ ) are shown in Figure 2. For ISM index, $f_{1}\left(x_{1} \mid Z_{t}=1\right)$ and $f_{1}\left(x_{1} \mid Z_{t}=0\right)$ get closer to each other when we predict recession at the longer horizon, indicating a weakening capacity 
of distinguishing two regimes defined by $Z_{t}$. For yield spread, these two densities remain roughly unchanged at both horizons. The dependence structure between the two predictors is captured by $\rho$ and $d f$. The correlation coefficient $\rho_{R}$ is negative, while $\rho_{E}$ is positive (both are significant). ${ }^{6}$ This pattern is also revealed in Figure 1, where both Figures 1(a) and 1(c) for $Z_{t}=1$ show the presence of negative relationship between the two predictors while their correlation reverses sign in Figures 1(b) and 1(d) for $Z_{t}=0$. Table 1 also reports two additional dependence measures: Kendall's tau and Spearman's rho, which are denoted by $\tau$ and rho respectively. Unlike the standard correlation coefficient $\rho$, these are computed based on ranks of two random variables, and hence are unaffected by the marginal distribution of each variable. For t-copula, we have $\rho=\sin \left(\frac{\pi}{2} \tau\right)$ and $\rho=2 \sin \left(\frac{\pi}{6}\right.$ rho $)$. Therefore, all three measures have an one-to-one relationship with each other, and they provide essentially the same information. As shown in this table, $\rho, \tau$ and rho share the identical sign and significance despite the differing magnitudes. The degrees of freedom when $Z_{t}=1$ is large, implying that the Gaussian copula serves as a good approximation and the tail dependence between two predictors should be quite weak. This is consistent with Figures 1(a) and 1(c), where the number of points that lie in the upper-right and lower-left corners is very small. However, this is not the case during the expansion months $\left(Z_{t}=0\right)$, and there are clearly more points in Figures 1(b) and 1(d) located in the corresponding regions. This can be thought of as a consequence of the positive tail dependence in the t-copula with moderate degrees of freedom, which is ruled out by any Gaussian copula.

ROC curves evaluated at the posterior means of $\theta$ are displayed in Figures 3(a) and 3(b). The dashed lines are based on (2) with a single predictor in $X_{t}$. The explicit analytic forms for these curves are available in Lahiri and Yang (2013a) for a binormal model. All other curves are approximated by simulation. To generate the blue solid lines (Lin ISM+YS), we refit the model by assuming a bivariate normal distribution and forcing two covariance matrices to be equal. As argued in Section 2, this amounts to using a linear combination scheme. We also tried the linear opinion pool in (7) with roughly the same results. ${ }^{7}$ ROC curves of the optimal non-linear combination scheme (2), Opt ISM+YS, are represented by green

\footnotetext{
${ }^{6}$ The $95 \%$ HPD intervals of $\rho_{R}-\rho_{E}$ are $[0.382,0.896]$ and $[0.352,0.745]$ for the 6 and 9-month-ahead forecasts respectively, indicating the differences between correlations across regimes are highly significant.

${ }^{7}$ Results based on (7) are available upon request.
} 
solid lines. Figures 3(a) and 3(b) show that the predictive power of ISM index deteriorates as horizon gets longer. The performance of yield spread, by contrast, is slightly better in predicting recessions 9 months ahead compared with 6 months ahead. These findings are consistent with the evidence in Figure 2. We will examine several determinants of forecast accuracy in our framework through a counterfactual exercise in Section 4.

The solid ROC curves based on combined forecasts uniformly dominate the dashed counterparts, implying that forecast combination, as an effective way to integrate the useful information contained in $\left(X_{1 t}, X_{2 t}\right)$, leads to substantial improvement in predictive ability over each predictor, particularly at the 6-month horizon. However YS individually performs better than ISM at the 9-month horizon, and is close to Lin ISM+YS. Although the linearly combined forecasts are never better than the optimally combined ones, they are overall close to each other. The difference between these two schemes depends on whether the bivariate normal distribution with homogeneous covariance matrix is a good approximation. For this particular example, it is reasonable to assume $\left(X_{1 t}, X_{2 t}\right)$ to be normally distributed when $Z_{t}=1$ but it does not seem to be valid when $Z_{t}=0$. Furthermore, virtually all of the second conditional moments in one regime are far away from their counterparts in the other regime, as shown in Table 1. For instance, $\left|\rho_{R}-\rho_{E}\right|=0.6$ and $\left|\sigma_{R, Y S}^{2}-\sigma_{E, Y S}^{2}\right|=1.6$ for 6-monthahead forecast. This provides a good explanation for the discernible gap between linear and non-linear schemes. As expected, the optimally combined forecasts perform the best for both horizons. For 6-month-ahead forecasts, each predictor contains useful information the other one does not and neither of them dominates the other over the entire range of the ROC curve. By exploiting independent information contained in both predictors, forecast combination is able to achieve a dramatic improvement over each single predictor. A different scenario occurs for the 9-month-ahead forecasts, in which the yield spread is significantly better than the diffusion index. Once the yield spread is known, the additional information provided by the diffusion index is marginally inconsequential. As a result, little gain is achieved through forecast combination over YS. In an extreme case, if the ISM index were completely random and as a result its ROC curve were the diagonal line, the combined forecast would have offered zero improvement over the yield spread since the information contained in the ISM index is redundant. 
Table 2 highlights the magnitude of improvement resulting from forecast combinations. The AUC value for each curve in Figure 3 is shown on the top of the panel of this table, and the percentage of improvement is on the bottom. On average, the AUC values of the combined forecasts are strikingly higher than those of the ISM diffusion index, and this considerable gain in discriminatory power is significant, as suggested by its 95\% HPD interval. However, the combined forecasts are not much better than the yield spread. For the 9-month-ahead forecasts, ImpvYS, which is defined as the proportionate improvement of the optimally combined forecast over the yield spread [i.e., ImpvYS $\equiv(A U C(O p t$ ISM+YS)-AUC(YS))/AUC(YS)], is only $3.9 \%$ because the marginal contribution from the diffusion index is found to be small. However, for the 6-month-ahead forecasts, this gain is $11.5 \%$ and significant.

As a single index to summarize the predictive accuracy of a forecasting system, AUC can be used to compare different forecasting systems. However, one is likely to miss important information by merely relying on AUC exclusively. For example, two ROC curves may cross at an interior point in the unit square with the same AUC value. On the left hand side of this point, one curve is higher than the other, which is reversed on the right hand side. By the AUC criterion, both curves are equally good. However, a decision maker having appetite for a higher hit rate compared to a lower false alarm rate will opt for the ROC curve, which is higher on the right hand side of the crossing point. Different decision makers may have different preferences. To fix the idea, we consider the linear score indexed by $m \in[0,1]$, i.e. $S(m)=m \zeta(\eta)+(1-m) *(1-\kappa(\eta))$. A higher $m$ means that the decision maker places more weight on $\zeta(\eta)$ than he puts on $1-\kappa(\eta)$. Given $m$, the problem faced by the decision maker is to choose a threshold value $\eta$ to maximize $S(m)$. We denote the maximizer and maximum value of this problem by $\eta^{*}(m)$ and $S^{*}(m)$, respectively. Both are functions of $m$. For a particular value of $m$, the decision maker may look at Figure 4 to appreciate how large the improvement in $S^{*}(m)$ can be by using the optimal non-linear scheme. In these graphs, the three lines trace out the relative improvements due to Opt ISM+YS over ISM, YS and Lin ISM+YS as $m$ varies over $[0,1]$. If $m$ is close to 0 or 1 , all models are seen to be roughly equivalent. When $m$ lies in the middle, say 0.5 , the non-linear combined forecasts offer a $17.5 \%(27.2 \%)$ improvement over the diffusion index while predicting recessions 6 (9) months ahead. Note that the score $S(0.5)$ is proportional to the Peirce skill score often 
used in the literature; See Manzato (2007) and Granger and Pesaran (2000). Over these $m$ values, at the 6-month horizon, the non-linear scheme offers $13 \%-18 \%$ improvement over ISM and YS. At the 9-month horizon, the non-linear scheme offers nearly $4 \%$ improvement over the linear scheme when $m$ is around 0.7 . Given that the latter is already a highly efficient classifier, this improvement can be considered economically significant. One implication is that the non-linear scheme seems to be a sensible choice for the target events that are relatively rare and undesirable like a financial meltdown, rare disease, tsunami, and the like. In these circumstances, the monetary losses associated with missed signals are certainly much larger than those due to false alarms.

To assess the parametric model outlined above in terms of its goodness-of-fit, we compute the posterior predictive p-values for a variety of statistics. Suppose $\tilde{X}_{T}$ is the another dataset of size $T$ generated from the model under study. We are able to draw a sample from $g\left(\tilde{X}_{T}\right) \mid X_{T}$ by sequentially simulating from $P\left(\theta \mid X_{T}\right)$ and $g\left(\tilde{X}_{T}\right) \mid \theta$, where $g(\cdot)$ contains some statistics of interest, $g\left(\tilde{X}_{T}\right) \mid X_{T}$ is the predictive distribution of $g\left(\tilde{X}_{T}\right)$ after observing $X_{T}$, and $g\left(\tilde{X}_{T}\right) \mid \theta$ is the likelihood function of $g\left(\tilde{X}_{T}\right)$. We can calculate the statistics $g\left(X_{T}\right)$ using the current sample $X_{T}$. If the model fits data well, $g\left(X_{T}\right)$ is fairly unlikely to lie too far away from the center of $g\left(\tilde{X}_{T}\right) \mid X_{T}$. The predictive $\mathrm{p}$-value is the relative frequency of those more extreme $g\left(\tilde{X}_{T}\right)$ (larger than $g\left(X_{T}\right)$ or small than $g\left(X_{T}\right)$ ). A small predictive p-value is taken as evidence against a model, and the rule-of-thumb is to reject a model when the p-value is below 0.05 . For our purpose, we select eight statistics in $g(\cdot)$ : the sample mean and variance for each predictor in each regime, with the results presented in Table 3. Confronted with the data, the t-copula with binormal margins is not rejected for all statistics. Indeed, the minimum p-value is 0.922 , meaning that the model is broadly consistent with the actual data.

Using (4), we can generate the probability of an impending recession, a probability that is of interest on its own right. Once the posterior distribution of $\theta$ is obtained and $P\left(Z_{t}=1\right)$ is estimated by $\sum_{t=1}^{T} Z_{t} / T$, the posterior behavior of $P\left(Z_{t}=1 \mid X_{1 t}, X_{2 t}\right)$ is also completely determined. In particular, we can get a path of the combined probability forecasts $\left\{P\left(Z_{t}=1 \mid X_{1 t}, X_{2 t}\right): t=1, \ldots, T\right\}$ for each horizon when the posterior mean of $\theta$ is plugged in. Since ISM and YS variables are never revised, except for the fact that the parameters are estimated using the whole sample, these generated probabilities of recessions can be considered 
to be real-time forecasts. The recession probabilities are presented in Figure 5, together with the forecasts based on each predictor. With a few exceptions, almost every recession since 1969 is accompanied by a higher-than-usual probability generated by the combined forecasts. The ISM diffusion index tends to generate high (low) probabilities 6 months ahead of recessions (expansions). However, it seems to be too conservative to give high probabilities of recessions 9 months ahead. Irrespective of which regime materializes, the ISM diffusion index always fluctuates around its average value, suggesting it lacks the ability to identify forthcoming economic recessions at the longer horizon. The yield spread is superior at the 9month horizon compared to 6-month horizon. The value of the combined forecasts are borne out remarkably well across the whole sample period with the linear and non-linear schemes, even though at the 9-month horizon YS performs very close to the combined forecasts. As we have mentioned before, the non-linear forecasts have a slight edge over those generated by the linear scheme.

\section{Counterfactual analysis}

To identify the determinants of accuracy gain through forecast combination, it is worthwhile to break down the overall improvement of the combined forecasts into several components. For the sake of brevity, we merely consider 6-month-ahead prediction in this section. Ideally, the forecasts should behave quite distinctly across regimes in order to have a high discriminatory power. Put it differently, the conditional distribution of the predictor given $Z_{t}=1$ must be strongly separated from that given $Z_{t}=0$. Not only does this require the two conditional means be different from each other but the conditional variances cannot be very high. Intu-

itively speaking, higher variance makes the two distributions overlap to a larger extent, and thereby dilutes a given mean difference. For instance, the AUC(YS) in Table 2 can be written as

$$
A U C(Y S)=\Phi\left(\frac{\mu_{R, Y S}-\mu_{E, Y S}}{\sqrt{\sigma_{R, Y S}^{2}+\sigma_{E, Y S}^{2}}}\right)
$$


for the binormal specification (cf. Krzanowski and Hand (2009)). The implication of (8) is that the ability of the yield spread in discriminating between two regimes, as measured by the AUC, depends positively on the difference between two conditional means $\mu_{R, Y S}-\mu_{E, Y S}$ but negatively on the magnitude of two conditional variances: $\sigma_{R, Y S}^{2}$ and $\sigma_{E, Y S}^{2}$. They correspond to two terms in the decomposition of the mean squared error between forecasts and actuals as suggested by Yates (1982). Given $\mu_{R, Y S}-\mu_{E, Y S}$, the minimum forecast variance is equal to $\left(\mu_{R, Y S}-\mu_{E, Y S}\right)^{2} P\left(Z_{t}=1\right) P\left(Z_{t}=0\right)$, which will only be achieved when the predictor takes $\mu_{R, Y S}$ on all occasions of $Z_{t}=1$, and it takes $\mu_{E, Y S}$ on other occasions. Under this circumstance, the variability of forecasts is completely due to the event's occurrence. Thus, the minimum variance is the smallest variance necessary to support a given wedge between $\mu_{R, Y S}$ and $\mu_{E, Y S}$. The actual variance that is beyond this minimum value is called excess variability, which is equal to $P\left(Z_{t}=1\right) \sigma_{R, Y S}^{2}+P\left(Z_{t}=0\right) \sigma_{E, Y S}^{2}$ and reflects how responsive the predictor is to information that is not related to the event's occurrence. When $\sigma_{R, Y S}^{2}=\sigma_{E, Y S}^{2}=0$, the excess variability becomes zero. To maximize the discriminatory power, a higher value of the minimum variance (hence a higher $\left|\mu_{R, Y S}-\mu_{E, Y S}\right|$ ) and a lower value of the excess variability (hence a lower $\sigma_{R, Y S}^{2}$ and $\sigma_{E, Y S}^{2}$ ) are desirable. In sum, a forecast with high discriminatory ability is expected to be highly sensitive to relevant information, but insensitive to irrelevant information related to the event's occurrence. In terms of the mean difference in Table 1, the yield spread is better than the ISM diffusion index. However, the diffusion index contributes because it has less excess variability compared to YS at the shorter horizon. Hence, these two predictors complement one another to produce more accurate forecasts, which absorbs the strength and abandons the weakness in each of them. In other words, the improvement of the combined forecast relative to any one predictor may stem from the marginal information in the other predictor.

As is obvious in Table 1, not only do the two conditional distributions for each predictor differ, but the dependence structure between the two predictors varies across regimes. The diffusion index is negatively correlated with the yield spread when $Z_{t}=1$ (recessions) but remarkably the correlation becomes positive when $Z_{t}=0$ (expansions). The combined forecast also benefits from the inclusion of this additional information regarding the dependence structure, which is not available for use by the single predictor or the linear scheme. The 
more distinct the values of $\rho_{R}$ and $\rho_{E}$ are, the higher is the improvement that can be made through non-linear forecast combination.

Figure 6 illustrates the sensitivity of ImpvISM and ImpvYS to the various determinants of the discriminatory ability. Specifically, it shows the quantitative importance of each of the factors that contributes towards the improved forecasting ability of the combined forecasts relative to ISM and YS individually. That is, it shows what happens to ImpvISM and ImpvYS when one of the parameters deviates from its posterior mean. Panel (a) of Figure 6 depicts how the relative superiority of the combined forecast in terms of AUC changes compared to ISM (ImpvISM) and to YS (ImpvYS) as the difference in the means of the conditional distributions of ISM diffusion index decreases from its observed value of 1.02 in the sample (see Table 1). This value is the right most point in Figure 6(a). As $\mu_{R, I S M}-\mu_{E, I S M}$ decreases (i.e., ISM is deteriorating as a classifier), the combined forecast also gets worse, but relatively less than the decline in that of ISM because YS continues to be the same. Thus, relatively speaking, the combined forecast improves upon ISM more because of YS. Simultaneously, the relative superiority of the combined forecast over YS decreases, albeit slowly, because YS by itself continues to be as good, and relatively speaking the combined forecast has less scope to improve upon YS. Figure 6(b) is a mirror image of Figure 6(a) as $\mu_{R, Y S}-\mu_{E, Y S}$ decreases to 0 from its sample value of 2.18. Note that the simulated changes in the mean differences of ISM and YS between the two regimes do not affect the distributions of YS and ISM nor their dependence structure. Figures 6(c) and 6(d) trace out the effects of changing the variances of ISM and YS respectively from their observed values. As the variance of ISM during recessions increases, the relative improvement of the combined forecast over ISM increases as a result of the deteriorating quality of ISM as an individual predictor with the quality of YS remaining the same. But ImpvYS seems to be largely insensitive to changes in $\sigma_{R, I S M}^{2}$. Figure $6(\mathrm{~d})$ that traces out the effect of changes in $\sigma_{R, Y S}^{2}$ is again a mirror image of Figure 6(c). The slopes of these curves are consistent with expectations, but their quantitative magnitudes are functions of the data structure and the forecast combination procedure.

Of particular interest is the role of changes in correlations between the two predictors as the regime changes. Figure 6(e) depicts ImpvISM and ImpvYS as $\left|\rho_{R}-\rho_{E}\right|$ increases. Note that $\left|\rho_{R}-\rho_{E}\right|=0.67$ in the sample. As this value goes from 0 to 1.2 , we find that the gain in 
the non-linear combination scheme over ISM and YS monotonically increases, as expected, in a parallel fashion. This gain is coming entirely from the increased value of the correlation during the two regimes with the individual predictors being the same. This affirms that the combined forecasts are able to explore the differential in dependence structure as well. When two conditional distributions of each predictor are fixed, more distinct correlations give rise to higher AUC(Opt ISM+YS), which further raises ImpvISM and ImpvYS. This is a noteworthy implication of our non-linear forecast combination framework.

The improvement of the non-linear scheme over the linear one is mainly attributed to three factors: the difference in conditional variances of ISM $\left(\left|\sigma_{R, I S M}^{2}-\sigma_{E, I S M}^{2}\right|\right)$, the difference in conditional variances of YS $\left(\left|\sigma_{R, Y S}^{2}-\sigma_{E, Y S}^{2}\right|\right)$, and the difference in correlation coefficients $\left|\rho_{R}-\rho_{E}\right|$. It is clear from (5) that the non-linear scheme differs from the linear one simply because of a non-zero $A_{2}$ under normality assumption. By definition, $A_{2}$ is determined by the difference in the second moments of the two predictors across regimes, that is, the difference in variances for each predictor and that in covariance between predictors. The larger these differences are, the more improvement the non-linear scheme would give. Figure 7 confirms that the gain from the non-linear scheme increases as each type of the difference gets larger. In our sample for 6-month-ahead forecasts, the difference in conditional variances of ISM was close to zero as compared to 1.60 for YS. As Figure 7 shows, the efficiency of the nonlinear scheme would have been greatly enhanced with small increase in $\left|\sigma_{R, I S M}^{2}-\sigma_{E, I S M}^{2}\right|$, compared to a similar increase in $\left|\sigma_{R, Y S}^{2}-\sigma_{E, Y S}^{2}\right|$, because the latter is already rather high. We also observe that had the difference in the correlation coefficients $\rho_{R}-\rho_{E}$ been more negative compared to its sample value of -0.60 , the non-linear scheme would have yielded a significant gain over the linear procedure. Thus, given a real dataset that exhibits sizeable differences in the second moments between two regimes, the proposed non-linear combination method can potentially be a much better option to pursue than the linear benchmark. 


\section{Conclusion}

This paper has proposed an optimal non-linear procedure to combine multiple predictors for a binary target event within a Bayesian framework. The resulting likelihood ratio rule, which has a solid rationale from Neyman-Pearson lemma, maximizes the hit rate for any given false alarm rate among all competing schemes. We show that under additional simplifying assumptions, the rule reduces to the linear combination scheme extensively used in practice. To illustrate the usefulness of this method, we use a binormal specification with t-copulas to characterize the marginal distributions and the contemporaneous dependence structure of the ISM new order diffusion index and the yield spread in predicting U.S. recessions defined by NBER. The merit of our approach is that the discriminatory ability of the individual predictors and the optimal combination rule can be uniquely determined by a few fundamental parameters, each of which controls a specific aspect of forecast skill. Evaluated at the posterior means of the estimated parameters, the aggregated forecasts have considerably higher AUC values than the individual predictors. Given that recessions are difficult to predict, this new approach offers a noteworthy improvement over the existing approaches. To better understand the underlying sources, we decomposed the overall improvement of the combined forecasts relative to each predictor into gains from using the differentials in the dependence structure of the two predictors in the two regimes, and gains from using the differentials in the means and variances of the conditional distributions of the other predictor. The two predictors seem to complement one another to produce a more accurate forecast, absorbing the strengths but abandoning the weaknesses in each of them. Moreover, the improvement of the non-linear scheme over the linear benchmark can be potentially large as long as the second moments of the two predictors behave quite distinctly between regimes.

Although the method presented in this paper is conceptually and computationally straightforward, it is subject to possible misspecification error. Its validity requires both the marginal distributions and the copulas be correctly specified. If a forecaster has a large training sample to estimate the model, we can circumvent this problem by using non-parametric or semiparametric methods. For example, if we want to maintain a parametric form for copulas, we 
may estimate the marginal distributions non-parametrically by kernel smoothing. The parameters in copulas are estimated by plugging in the non-parametric estimates. Asymptotic properties of this semi-parametric two-step estimator have been established in Chen et al. (2010). If and how the existing results can be modified to make inference on the resulting ROC curve is still an open question. Generalization of our combination scheme along this line would be a promising topic for future research. 


\section{Tables and Figures}

Table 1: Posterior summary of $\theta$

\begin{tabular}{|c|c|c|c|c|c|c|}
\hline \multirow[b]{2}{*}{$\theta$} & \multicolumn{3}{|c|}{6 months ahead } & \multicolumn{3}{|c|}{9 months ahead } \\
\hline & Mean & LHPD & UHPD & Mean & LHPD & UHPD \\
\hline$\mu_{R, I S M}$ & 0.865 & 0.415 & 1.357 & 0.510 & 0.077 & 0.941 \\
\hline$\mu_{E, I S M}$ & -0.153 & -0.371 & 0.082 & -0.096 & -0.350 & 0.194 \\
\hline$\mu_{R, Y S}$ & 0.890 & -0.684 & 2.309 & 1.076 & -0.256 & 2.374 \\
\hline$\mu_{E, Y S}$ & -1.294 & -1.555 & -0.980 & -1.310 & -1.794 & -0.848 \\
\hline$\sigma_{R, I S M}^{2}$ & 0.886 & 0.481 & 1.557 & 0.734 & 0.408 & 1.154 \\
\hline$\sigma_{E, I S M}^{2}$ & 0.902 & 0.626 & 1.219 & 1.026 & 0.657 & 1.446 \\
\hline$\sigma_{R, Y S}^{2}$ & 3.651 & 2.384 & 5.492 & 3.308 & 0.396 & 13.120 \\
\hline$\sigma_{E, Y S}^{2}$ & 2.051 & 1.202 & 3.105 & 1.967 & 1.346 & 2.675 \\
\hline$\rho_{R}$ & -0.398 & -0.624 & -0.181 & -0.247 & -0.258 & -0.234 \\
\hline$\rho_{E}$ & 0.272 & 0.130 & 0.441 & 0.306 & 0.104 & 0.495 \\
\hline$\tau_{R}$ & -0.260 & -0.408 & -0.097 & -0.159 & -0.166 & -0.150 \\
\hline$\tau_{E}$ & 0.175 & 0.083 & 0.291 & 0.198 & 0.066 & 0.330 \\
\hline$r h o_{R}$ & -0.382 & -0.606 & -0.174 & -0.237 & -0.248 & -0.224 \\
\hline$r h o_{E}$ & 0.260 & 0.124 & 0.424 & 0.293 & 0.099 & 0.478 \\
\hline$d f_{R}$ & 8008.878 & 7942.348 & 8085.989 & 27392.215 & 26900.526 & 27899.045 \\
\hline$d f_{E}$ & 70.974 & 65.942 & 75.709 & 28.565 & 11.829 & 53.228 \\
\hline
\end{tabular}


Table 2: Comparison of posterior AUC

\begin{tabular}{ccccccccc}
\hline & \multicolumn{3}{c}{6 months ahead } & & \multicolumn{3}{c}{9 months ahead } \\
\cline { 8 - 9 } Object & Mean & LHPD & UHPD & & Mean & LHPD & UHPD \\
\hline AUC(ISM) & 0.777 & 0.671 & 0.887 & & 0.676 & 0.535 & 0.803 \\
AUC(YS) & 0.820 & 0.613 & 0.956 & & 0.851 & 0.705 & 0.958 \\
AUC(Lin ISM+YS) & 0.891 & 0.738 & 0.978 & 0.862 & 0.706 & 0.942 \\
AUC(Opt ISM+YS) & 0.914 & 0.835 & 0.983 & & 0.884 & 0.808 & 0.966 \\
\hline ImpvISM & 0.176 & 0.008 & 0.381 & & 0.307 & 0.087 & 0.664 \\
ImpvYS & 0.115 & 0.011 & 0.386 & & 0.039 & 0.002 & 0.209 \\
\hline
\end{tabular}

Notes: The top panel of the table contains the AUC values computed for each curve in Figure 3. ImpvISM=(AUC(Opt ISM+YS)-AUC(ISM))/AUC(ISM), and ImpvYS=(AUC(Opt ISM+YS)-AUC(YS))/AUC(YS).

Table 3: Model comparison in terms of the posterior predictive p-values

\begin{tabular}{ccc}
\hline$g(\cdot)$ & 6 months ahead & 9 months ahead \\
\hline$p\left(\mu_{R, I S M}\right)$ & 0.984 & 0.956 \\
$p\left(\sigma_{R, I S M}^{2}\right)$ & 0.980 & 0.970 \\
$p\left(\mu_{E, I S M}\right)$ & 0.984 & 0.988 \\
$p\left(\sigma_{E, I S M}^{2}\right)$ & 0.994 & 0.922 \\
$p\left(\mu_{R, Y S}\right)$ & 0.974 & 0.986 \\
$p\left(\sigma_{R, Y S}^{2}\right)$ & 0.996 & 0.976 \\
$p\left(\mu_{E, Y S}\right)$ & 0.984 & 0.970 \\
$p\left(\sigma_{E, Y S}^{2}\right)$ & 0.956 & 0.994 \\
\hline
\end{tabular}

Notes: $p\left(\mu_{R, I S M}\right)$ is the posterior predictive p-value when $\mu_{R, I S M}$ is the statistic of interest. Other p-values in this table are similarly defined. 
Figure 1: Scatter plots of $F_{1}\left(X_{1 t} \mid Z_{t}\right)$ and $F_{2}\left(X_{2 t} \mid Z_{t}\right)$

(a) 6 months ahead $\left(Z_{t}=1\right)$

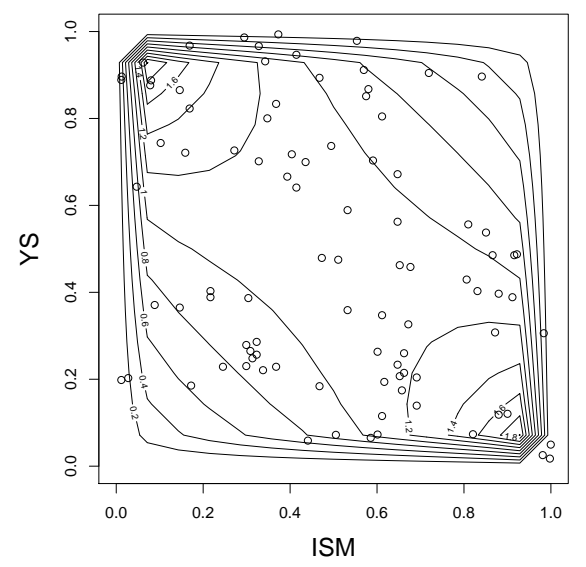

(c) 9 months ahead $\left(Z_{t}=1\right)$

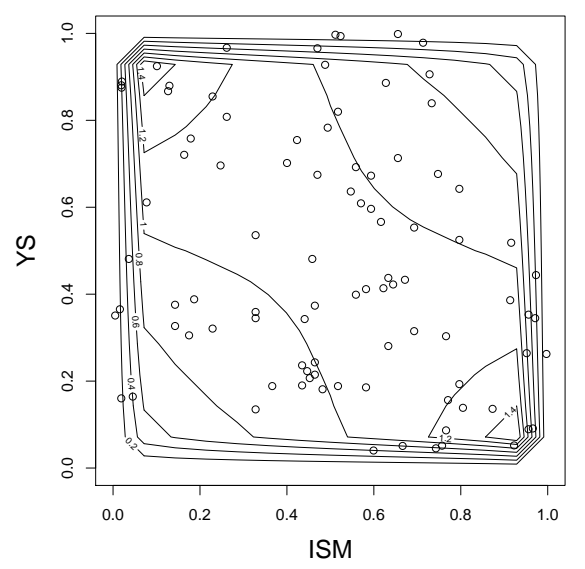

(b) 6 months ahead $\left(Z_{t}=0\right)$

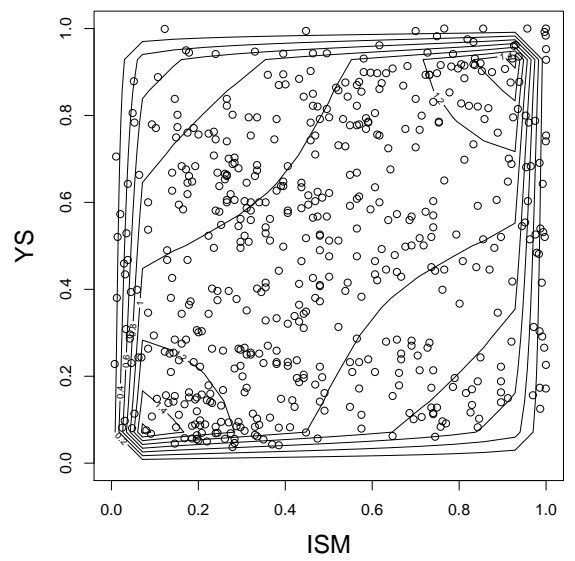

(d) 9 months ahead $\left(Z_{t}=0\right)$

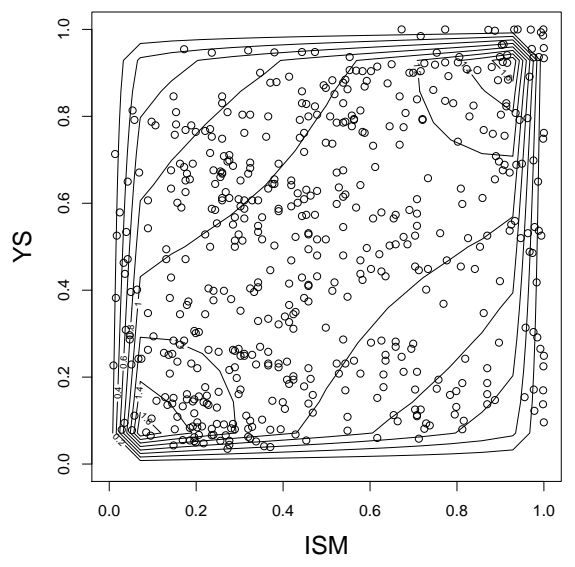

Note: The solid curves represent the contours of the fitted t-copulas. 
Figure 2: Conditional densities of two predictors

(a) 6 months ahead (ISM)

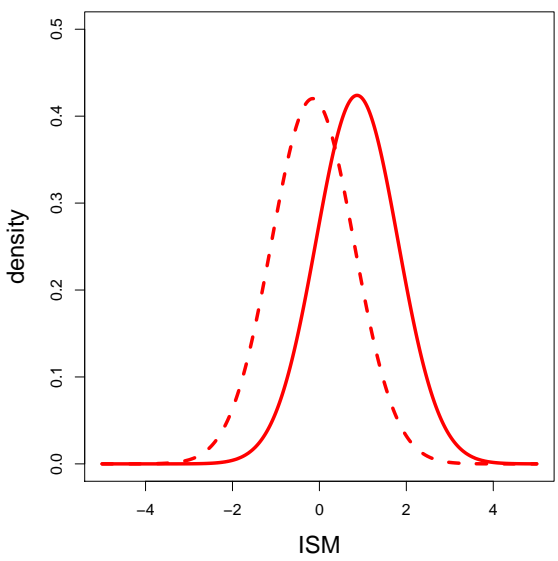

(c) 6 months ahead (YS)

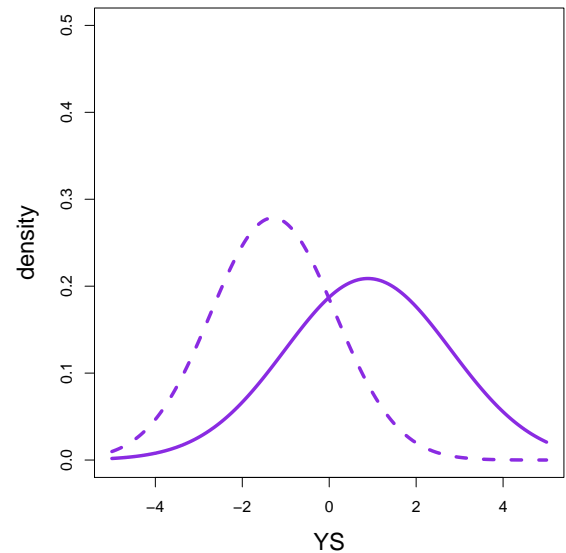

(b) 9 months ahead (ISM)

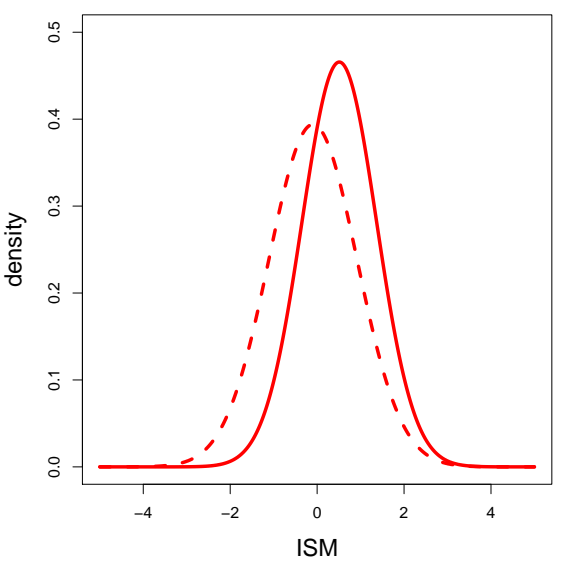

(d) 9 months ahead (YS)

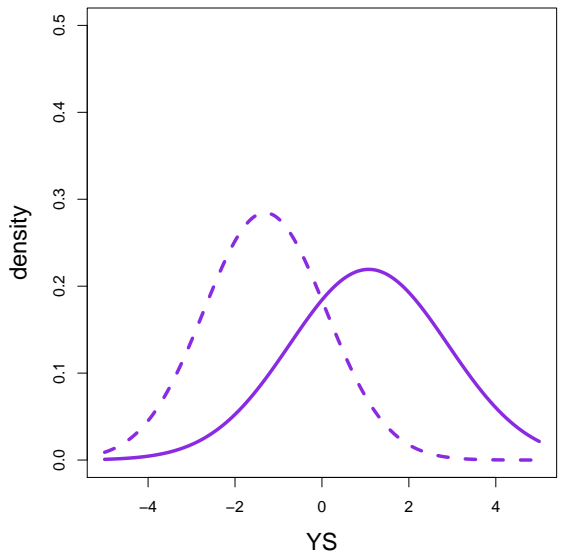

Note: The solid curve represents the conditional density given $Z_{t}=1$ and the dotted curve is the conditional density given $Z_{t}=0$. 
Figure 3: ROC curves evaluated at posterior means

(a) 6 months ahead

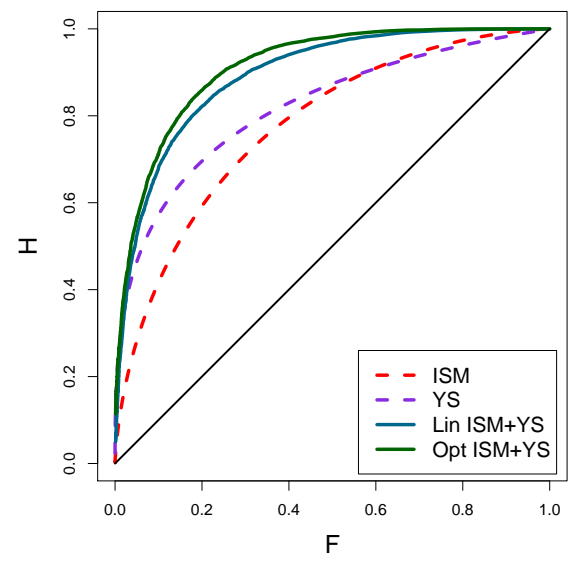

(b) 9 months ahead

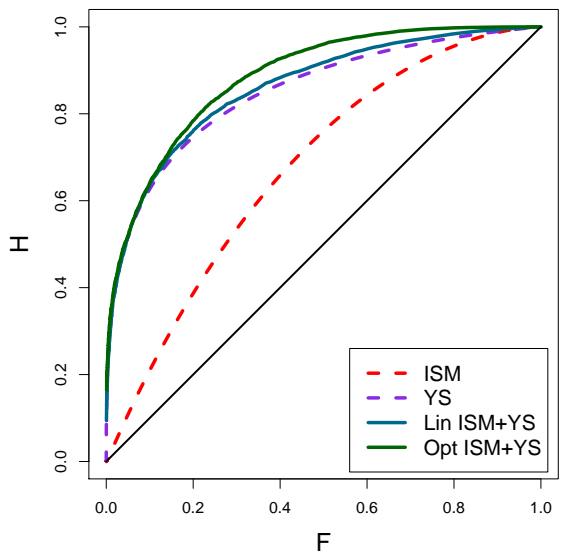

Notes: The vertical axis " $\mathrm{H}$ " is the hit rate, while the horizontal axis " $\mathrm{F}$ " is the false alarm rate. "Lin ISM+YS" and "Opt ISM+YS" are the ROC curves of the linearly and optimally combined non-linear forecasts respectively.

Figure 4: Improvements in the optimally combined forecast in terms of the linear score

(a) 6 months ahead

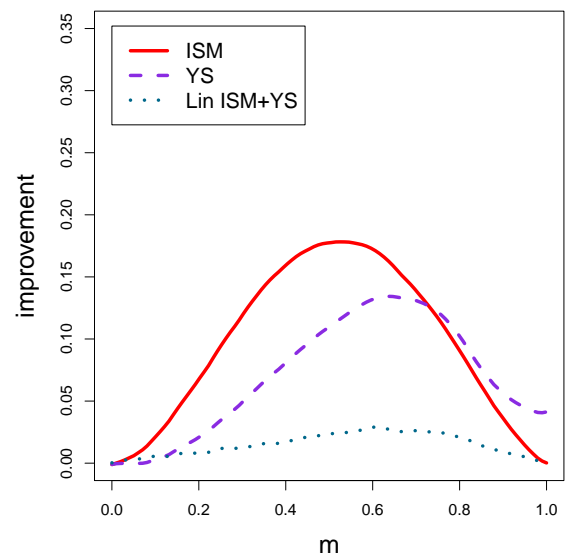

(b) 9 months ahead

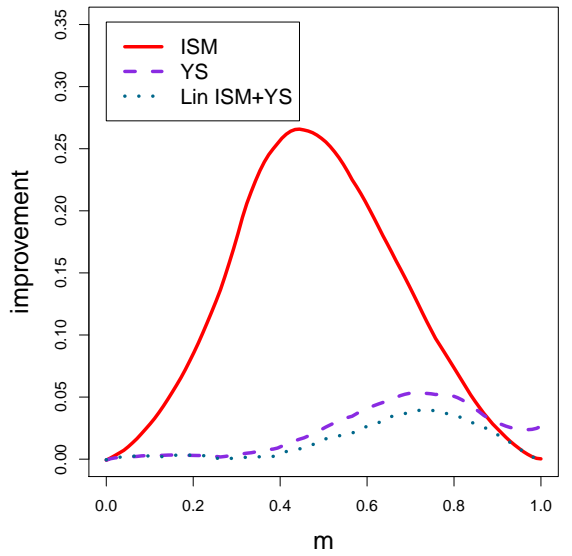

Notes: "ISM" represents the improvement of the optimally combined forecast over ISM diffusion index, that is, $\left(S^{*}(m)(\mathrm{Opt}\right.$ ISM+YS)- $\left.S^{*}(m)(\mathrm{ISM})\right) / S^{*}(m)(\mathrm{ISM})$. All of the other curves are calculated in the same way. 

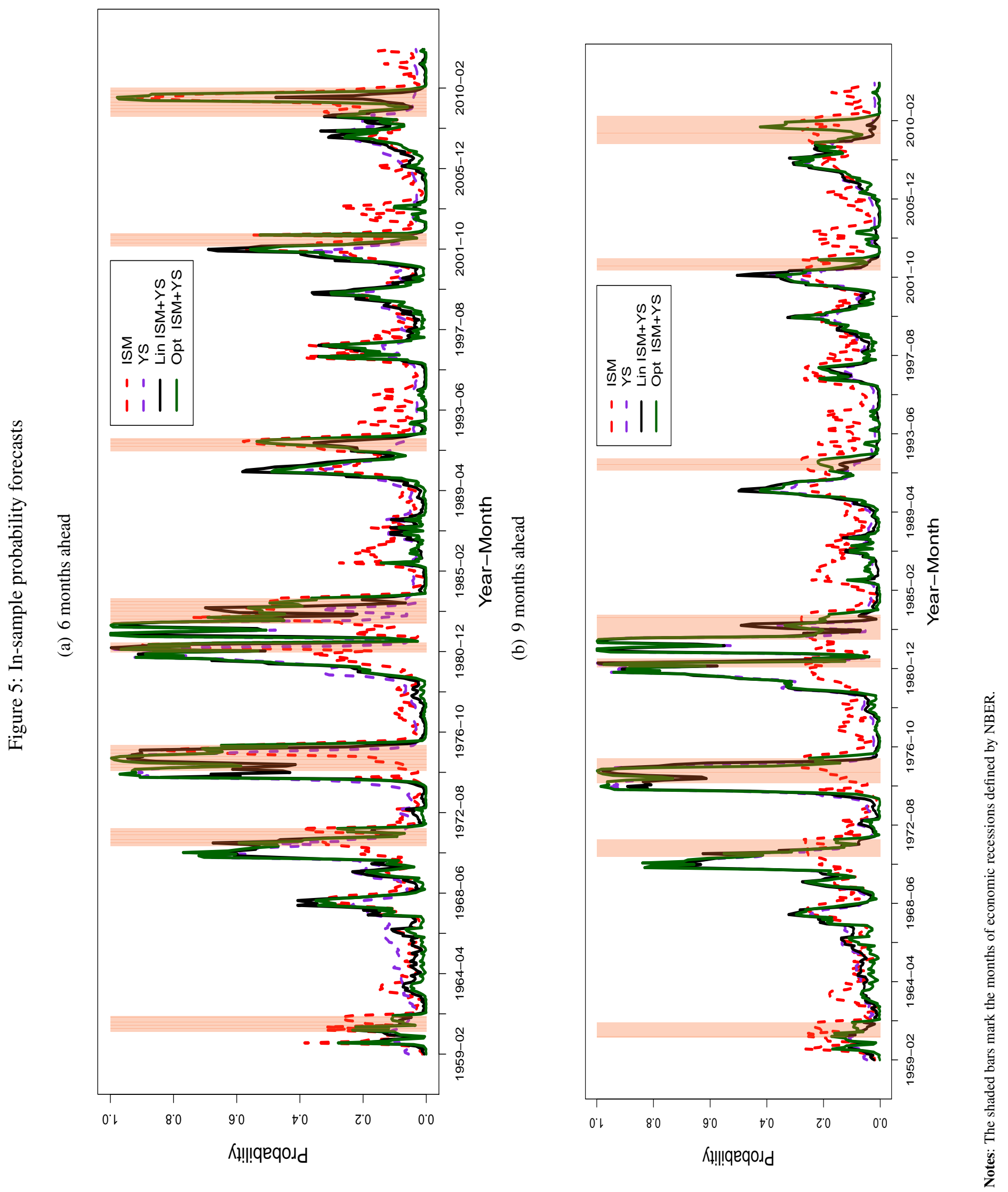
Figure 6: Determinants of improvement in the combined forecasts over ISM and YS

(a) Mean difference (ISM)

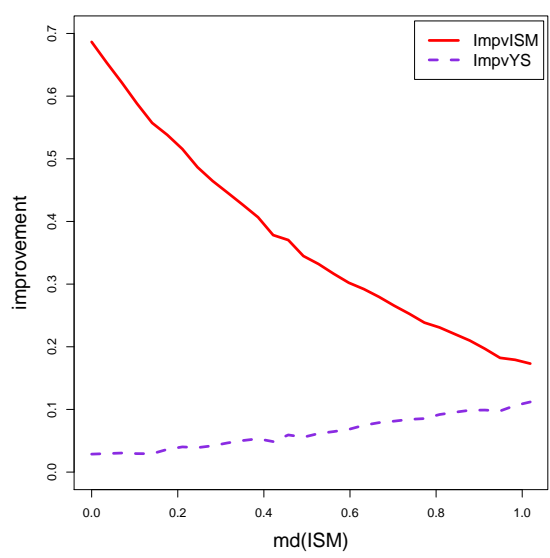

(c) Variance (ISM)

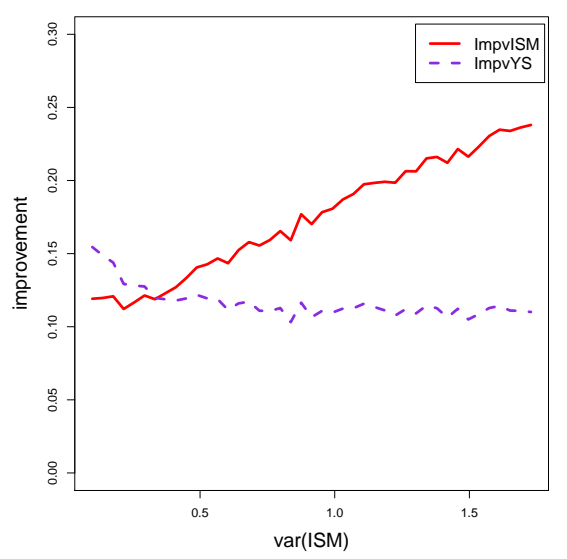

(b) Mean difference (YS)

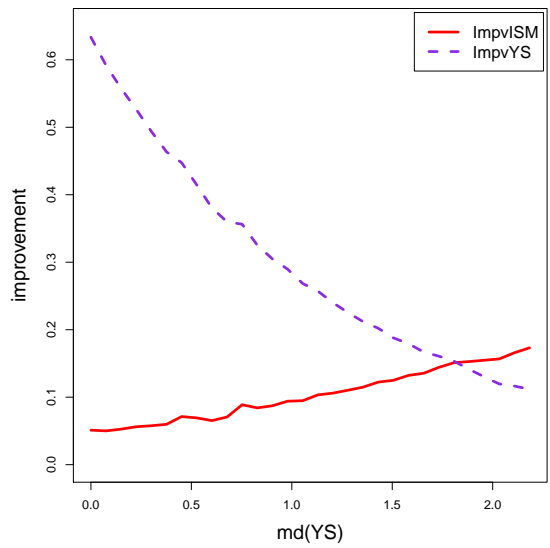

(d) Variance (YS)

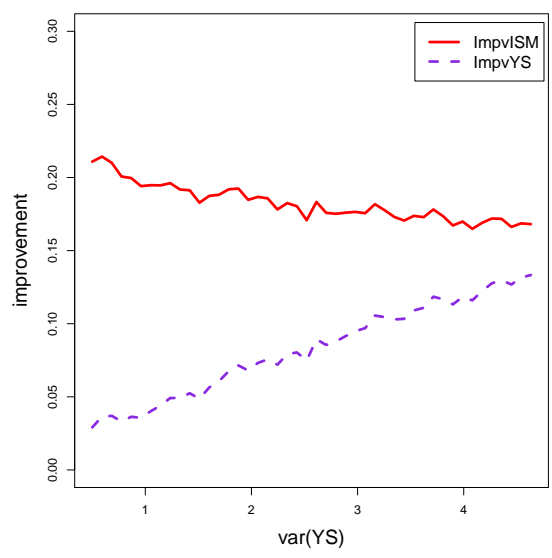

(e) Correlation difference

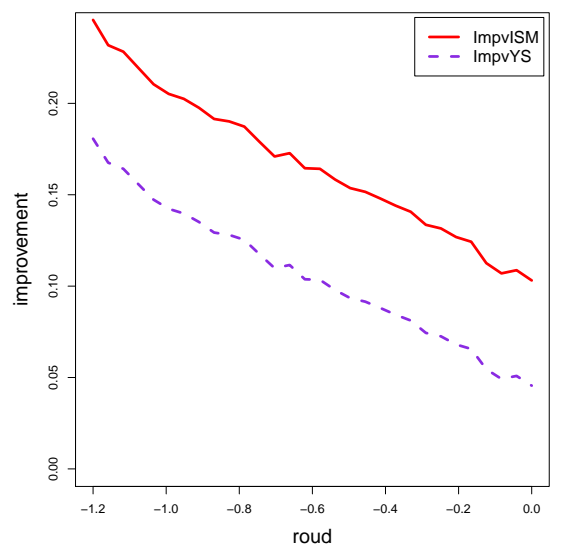

Notes: For the ISM, md(ISM) $=\mu_{R, I S M}-\mu_{E, I S M}$ and $\operatorname{var}(\mathrm{ISM})=\sigma_{R, I S M}^{2}$. The similar notations apply for the yield spread. roud $=\rho_{R}-\rho_{E}$. 


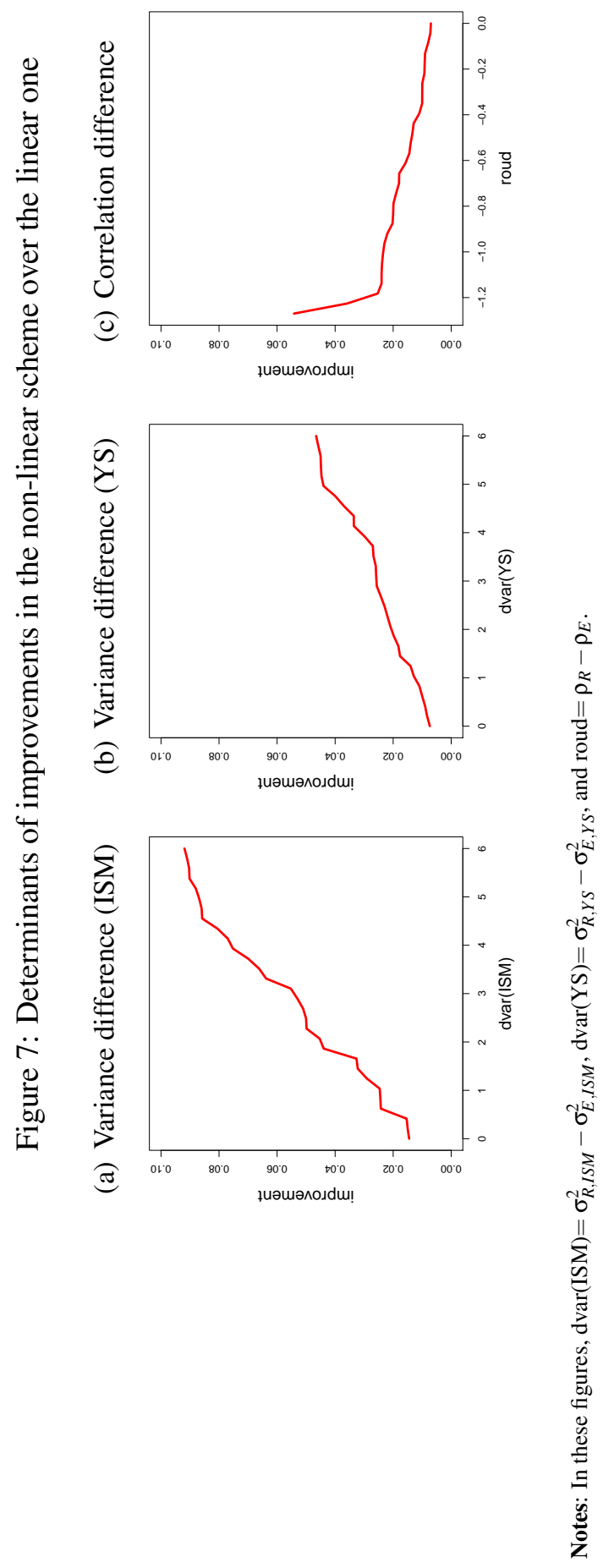




\section{References}

Albert, J. (2009), Bayesian Computation with R, Springer.

Anatolyev, S. (2009), 'Multi-Market Direction-of-Change Modeling Using Dependence Ratios', Studies in Nonlinear Dynamics \& Econometrics 13, Article 5.

Andrews, D. W. K. (1991), 'Heteroskedasticity and Autocorrelation Consistent Covariance Matrix Estimation', Econometrica 59, 817-858.

Andrews, D. W. K. and Monahan, J. C. (1992), 'An Improved Heteroskedasticity and Autocorrelation Consistent Covariance Matrix Estimator', Econometrica 60, 953-966.

Bates, J. M. and Granger, C. W. J. (1969), 'The Combination of Forecasts', Operational Research Quarterly 20, 451-468.

Berge, T. J. and Jordà, Ò. (2011), 'Evaluating the Classification of Economic Activity into Recessions and Expansions', American Economic Journal: Macroeconomics 3, 246-277.

Berk, R. H. (1966), 'Limiting Behavior of Posterior Distributions when the Model is Incorrect', The Annals of Mathematical Statistics 37, 51-58.

Breymann, W., Dias, A. and Embrechts, P. (2003), 'Dependence Structures for Multivariate High-Frequency Data in Finance', Quantitative Finance 3, 1-14.

Brier, G. W. (1950), 'Verification of Forecasts Expressed in Terms of Probability', Monthly Weather Review 78, 1-3.

Bunke, O. and Milhaud, X. (1998), 'Asymptotic Behavior of Bayes Estimates under Possibly Incorrect Models', The Annals of Statistics 26, 617-644.

Chen, X., Fan, Y., Pouzo, D. and Ying, Z. (2010), 'Estimation and Model Selection of Semiparametric Multivariate Survival Functions under General Censorship', Journal of Econometrics 157, 129-142. 
Dawid, A. P. (1984), 'Present Position and Potential Developments: Some Personal Views: Statistical Theory: The Prequential Approach', Journal of the Royal Statistical Society, Series A 147, 278-292.

De Luca, G. and Carfora, A. (2014), 'Predicting U.S. Recessions through a Combination of Probability Forecasts', Empirical Economics 46, 127-144.

Demarta, S. and McNeil, A. J. (2005), 'The t Copula and Related Copulas', International Statistical Review 73, 111-129.

Drehmann, M. and Juselius, M. (2012), Improving Early Warning Indicators for Banking Crises Satisfying Policy Requirements. Paper presentation at: Understanding Macroprudential Regulation, Norges Bank, Oslo, 2930 November 2012.

Egan, J. P. (1975), Signal Detection Theory and ROC Analysis, New York: Academic Press.

Estrella, A. and Mishkin, F. S. (1996), 'The Yield Curve as a Predictor of U.S. Recessions', Current Issues in Economics and Finance 2, 41-51.

Estrella, A. and Mishkin, F. S. (1998), 'Predicting U.S. Recessions: Financial Variables as Leading Indicators', The Review of Economics and Statistics 80, 45-61.

Fawcett, T. (2006), ‘An Introduction to ROC Analysis', Pattern Recognition Letters 27, 861874.

Geweke, J. (2005), Contemporary Bayesian Econometrics and Statistics, John Wiley \& Sons.

Gneiting, T. and Raftery, A. E. (2007), 'Strictly Proper Scoring Rules, Prediction, and Estimation', Journal of the American Statistical Association 102, 359-378.

Graham, J. R. (1996), ‘Is a Group of Economists Better Than One? Than None?', The Journal of Business 69, 193-232.

Granger, C. W. J. and Pesaran, M. H. (2000), 'Economic and Statistical Measures of Forecast Accuracy', Journal of Forecasting 19, 537-560. 
Granger, C. W. J. and Ramanathan, R. (1984), 'Improved Methods of Combining Forecasts', Journal of Forecasting 3, 197-204.

Green, D. M. and Swets, J. A. (1966), Signal Detection Theory and Psychophysics, Wiley: New York.

Hastie, T., Tibshirani, R. and Friedman, J. (2001), The Elements of Statistical Learning: Data Mining, Inference, and Prediction, Springer.

Kamstra, M. and Kennedy, P. (1998), 'Combining Qualitative Forecasts Using Logit', International Journal of Forecasting 14, 83-93.

Koop, G. (2003), Bayesian Econometrics, John Wiley \& Sons.

Krzanowski, W. J. and Hand, D. J. (2009), ROC Curves for Continuous Data, Chapman \& Hall.

Lahiri, K. and Wang, J. G. (1996), Interest Rate Spreads as Predictors of Business Cycles, in G. S. Maddala and C. R. Rao, eds, 'Handbook of Statistics 14 (Statistical Methods in Finance)', North-Holland Amsterdam, pp. 297-315.

Lahiri, K. and Wang, J. G. (1994), 'Predicting Cyclical Turning Points with Leading Index in a Markov Switching Model', Journal of Forecasting 13, 245-263.

Lahiri, K. and Wang, J. G. (2013), 'Evaluating Probability Forecasts for GDP Declines using Alternative Methodologies', International Journal of Forecasting 29, 175-190.

Lahiri, K. and Yang, L. (2013a), Confidence Bands for ROC Curves with Serially Dependent Data. Working paper, Department of Economics, State University of New York at Albany.

Lahiri, K. and Yang, L. (2013b), Forecasting Binary Outcomes, in A. Timmermann and G. Elliott, eds, 'Handbook of Economic Forecasting Volume 2B', North-Holland Amsterdam, pp. 1025-1106.

Levanon, G., Ozyildirim, A., Schaitkin, B. and Zabinska, J. (2011), Comprehensive Benchmark Revisions for The Conference Board Leading Economic Index for the United States. EPWP 11-06, The Conference Board, New York, December 2011. 
Manzato, A. (2007), 'A Note On the Maximum Peirce Skill Score', Weather and Forecasting 22, 1148-1154.

Mashal, R., Naldi, M. and Zeevi, A. (2003), 'On the Dependence of Equity and Asset Returns', Risk 16, 83-87.

McIntosh, M. W. and Pepe, M. S. (2002), 'Combining Several Screening Tests: Optimality of the Risk Score', Biometrics 58, 657-664.

Müller, U. K. (2013), 'Risk of Bayesian Inference in Misspecified Models, and the Sandwich Covariance Matrix', Econometrica 81, 1805-1849.

Nelsen, R. B. (2006), An Introduction to Copulas, Springer.

Newey, W. K. and West, K. D. (1987), ‘A Simple, Positive Semi-definite, Heteroskedasticity and Autocorrelation Consistent Covariance Matrix', Econometrica 55, 703-708.

Neyman, J. and Pearson, E. S. (1933), 'On the Problem of the Most Efficient Tests of Statistical Hypothesis', Philosophical Transactions of the Royal Society of London, Series A 231, 289-337.

Patton, A. J. (2006), 'Modelling Asymmetric Exchange Rate Dependence', International Economic Review 47, 527-556.

Patton, A. J. (2012), 'A Review of Copula Models for Economic Time Series', Journal of Multivariate Analysis 110, 4-18.

Patton, A. J. (2013), Copula Methods for Forecasting Multivariate Time Series, in A. Timmermann and G. Elliott, eds, 'Handbook of Economic Forecasting Volume 2B', NorthHolland Amsterdam, pp. 899-960.

Pepe, M. S. (2003), The Statistical Evaluation of Medical Tests for Classification and Prediction, Oxford University Press.

Ramsey, J. B. (1969), 'Tests for Specification Errors in Classical Linear Least-Squares Regression Analysis', Journal of the Royal Statistical Society, Series B 31, 350-371. 
Ranjan, R. and Gneiting, T. (2010), 'Combining Probability Forecasts', Journal of the Royal Statistical Society, Series B 72, 71-91.

Schervish, M. J. (1989), 'A General Method for Comparing Probability Assessors', Annals of Statistics 17, 1856-1879.

Scotti, C. (2011), 'A Bivariate Model of Federal Reserve and ECB Main Policy Rates', International Journal of Central Banking 7, 37-78.

Stephenson, D. B. (2000), 'Use of the 'Odds Ratio' for Diagnosing Forecast Skill', Weather Forecasting 15, 221-232.

Swets, J. A., Dawes, R. M. and Monahan, J. (2000), 'Better Decisions through Science', Scientific American 283, 82-87.

Timmermann, A. (2006), Forecast Combinations, in G. Elliott, C. W. J. Granger and A. Timmermann, eds, 'Handbook of Economic Forecasting', North-Holland Amsterdam, pp. 135196.

Trivedi, P. K. and Zimmer, D. M. (2005), 'Copula Modeling: An Introduction for Practitioners', Foundations and Trends in Econometrics 1, 1-111.

White, H. (1982), 'Maximum Likelihood Estimation of Misspecified Models', Econometrica 50, $1-25$.

Yates, J. F. (1982), 'External Correspondence: Decompositions of the Mean Probability Score', Organizational Behavior and Human Performance 30, 132-156.

Zhou, X. H., Obuchowski, N. A. and McClish, D. K. (2002), Statistical Methods in Diagnostic Medicine, John Wiley \& Sons. 